\title{
Numerical Continuation of Symmetric Periodic Orbits
}

\author{
Claudia Wulff \\ Department of Mathematics \& Statistics \\ University of Surrey \\ Guildford GU2 7XH, United Kingdom \\ email: c.wulff@surrey.ac.uk
}

and

Andreas Schebesch

Fachbereich Mathematik und Informatik

Freie Universität Berlin

14195 Berlin, Germany

email: schebesc@inf.fu-berlin.de

Dedicated to the memory of our dear friend and colleague Karin Gatermann

\begin{abstract}
The bifurcation theory and numerics of periodic orbits of general dynamical systems is well developed, and in recent years there has been rapid progress in the development of a bifurcation theory for symmetric dynamical systems. But there are hardly any results on the numerical computation of those bifurcations yet. In this paper we show how spatiotemporal symmetries of periodic orbits can be exploited numerically. We describe methods for the computation of symmetry breaking bifurcations of periodic orbits for free group actions and show how bifurcations increasing the spatiotemporal symmetry of periodic orbits (including period halving bifurcations and equivariant Hopf bifurcations) can be detected and computed numerically. Our pathfollowing algorithm is based on a multiple shooting algorithm for the numerical computation of periodic orbits via an adaptive Poincaré section and a tangential continuation method with implicit reparametrization.
\end{abstract}

AMS subject classification. 37G15, 37G40, 37M20, 65P30

Keywords. Numerical continuation, symmetry breaking bifurcations, symmetric periodic orbits 


\section{Contents}

1 Introduction 3

2 Continuation of symmetric periodic orbits 4

2.1 Computation of single periodic orbits - single shooting method . . . . . . . . . 4

2.2 Symmetries of periodic orbits and how to exploit them . . . . . . . . . . . 6

2.3 Multiple shooting approach . . . . . . . . . . . . . . . . . 8

2.4 Continuation of nondegenerate periodic orbits . . . . . . . . . . . . . . . . 10

2.4.1 Single shooting method . . . . . . . . . . . . . . . . 10

2.4 .2 Multiple shooting ansatz . . . . . . . . . . . . . . . . 11

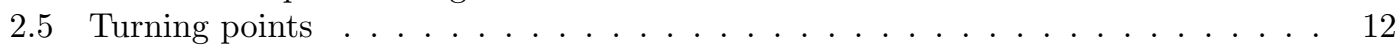

3 Computation of flip down and flip up bifurcations $\quad 12$

3.1 Detection and computation of period doubling bifurcations . . . . . . . . . . 13

3.1.1 Numerical detection of period doubling bifurcations . . . . . . . . . . . 14

3.1.2 Computation of period doubling bifurcation points . . . . . . . . . . . . 14

3.1.3 Computation of start off directions for the bifurcating branch . . . . . . . 15

3.2 Detection and computation of period halving bifurcations . . . . . . . . . . . 16

3.2.1 Detection of period halving bifurcations . . . . . . . . . . . . 16

3.2.2 Computation of period halving bifurcation points and start off directions 17

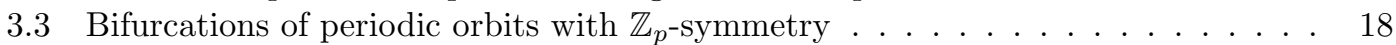

3.3.1 Flip pitchfork bifurcation . . . . . . . . . . . . . . . 18

3.3.2 Flip doubling bifurcation . . . . . . . . . . . . . . . . . 19

3.4 Numerical computation of symmetry breaking bifurcations . . . . . . . . . . . . . 19

3.4.1 Detection and computation of flip down bifurcations . . . . . . . . . . . 19

3.4 .2 Initialization of the bifurcating branch . . . . . . . . . . . . . . . 19

3.5 Numerical computation of symmetry increasing bifurcations . . . . . . . . . . . 20

3.5.1 Detection of flip up bifurcations . . . . . . . . . . . . . . . 20

3.5.2 Computation of flip up points and start off tangents . . . . . . . . . . . 21

4 Computation of equivariant Hopf points $\quad 21$

4.1 Hopf bifurcations for non-symmetric systems . . . . . . . . . . . . . . 21

4.1.1 Detection of Hopf points along branches of periodic orbits . . . . . . . . 22

4.1.2 Computation of Hopf bifurcations of non-symmetric systems . . . . . . . 22

4.2 Detection and computation of equivariant Hopf points . . . . . . . . . . . . . 24

4.2.1 Equivariant Hopf points . . . . . . . . . . . . . . . . . 24

4.2.2 Detection of equivariant Hopf points . . . . . . . . . . . . . . . 25

4.2.3 Computation of equivariant Hopf bifurcations . . . . . . . . . . . . . . 25

5 Applications $\quad 31$

5.1 The Lorenz model - comparison with AUTO and CONTENT . . . . . . . . . . 31

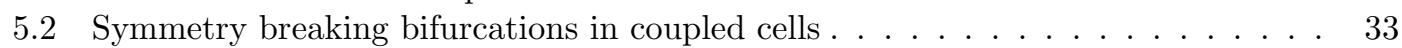

5.3 Electronic ring oscillator . . . . . . . . . . . . . . . . . . . 35

$\begin{array}{lr}\text { Conclusion } & 39\end{array}$ 


\section{Introduction}

The bifurcation theory and numerics of periodic orbits of general dynamical systems is well developed, see e.g. $[1,8,13,16,17]$. Frequently the considered problems possess certain symmetries. Symmetries change the generic behaviour of a dynamical system dramatically, and in recent years there has been rapid progress in the development of a bifurcation theory for periodic orbits of symmetric dynamical systems, see eg $[9,11,18,19,24]$. But there are hardly any results on the numerical computation of those bifurcations yet. Gatermann and Hohmann [10] developed numerical methods for the exploitation of symmetry and the computation of symmetry breaking and symmetry increasing bifurcations of stationary solutions and implemented those methods in the mixed symbolic numerical code SYMCON. They treat finite symmetry groups. Cliffe et al [2] developed numerical methods for the computation of bifurcations of stationary solutions in the case of continuous rotational symmetries.

In this paper we start a systematic theory on numerical bifurcation of symmetric periodic orbits by extending the methods of Gatermann and Hohmann [10] to periodic solutions. We consider a parameter dependent dynamical system

$$
\dot{x}=f(x, \lambda), \quad f: \mathbb{R}^{n} \times \mathbb{R} \rightarrow \mathbb{R}^{n},
$$

which is equivariant with respect to a finite symmetry group $\Gamma \subset \operatorname{GL}(n)$, i.e.,

$$
\gamma f(x, \lambda)=f(\gamma x, \lambda) \quad \forall \gamma \in \Gamma, x \in \mathbb{R}^{n}, \lambda \in \mathbb{R} .
$$

In most parts of this paper we assume that $\Gamma$ acts freely, i.e., $\gamma x \neq x$ for all $\gamma \neq$ id and $x \in \mathbb{R}^{n}$. We numerically continue periodic solutions with respect to the parameter $\lambda$ exploiting possible symmetries and computing symmetry breaking and symmetry increasing bifurcations of those periodic orbits. For the computation of periodic orbits we employ the multiple shooting algorithm presented by Deuflhard [5], which we briefly recall in Section 2.1. Section 2.2 is concerned with the exploitation of spatial and spatio-temporal symmetries of periodic orbits in the multiple shooting context. In Section 2.4 the aspect of continuation is added and the pathfollowing method of Deuflhard, Fiedler and Kunkel [7] is used for the continuation of periodic solutions in the single and multiple shooting approach. A different method for the numerical continuation of periodic solutions with symmetry based on Fourier expansions has been presented by Dellnitz [4].

In Sections 3 and 4 bifurcations of symmetric periodic orbits are treated. First symmetry breaking and symmetry increasing bifurcations of periodic orbits are considered (Section 3) and then equivariant Hopf bifurcations along periodic orbits (Section 4). In these sections numerical techniques of Gatermann and Hohmann [10] are extended from stationary solutions to periodic solutions.

Generic symmetry breaking bifurcations of periodic orbits of free group actions correspond to period doubling bifurcations in the space of group orbits, or equivalently, to period doubling bifurcations of the symmetry reduced Poincaré map [18]. In Section 3.4 we show how such symmetry breaking bifurcations can be detected and computed numerically by extending the corresponding methods for non-symmetric systems, c.f. Section 3.1.

We have also developed methods for the computation of bifurcations which increase the spatio-temporal symmetry of the periodic solution, in particular an algorithm for the computation of period halving points which is based on the methods for computing period doubling bifurcation points, see Sections 3.2 and 3.5.

In Section 4 we derive methods for the detection of equivariant Hopf points along branches of symmetric periodic orbits and present an extended system for the computation of equivariant 
Hopf points. The main problem is how to deal with multiple Hopf eigenvalues forced by symmetry. The issue of numerically dealing with multiple critical eigenvalues was treated by Cliffe et al [2] in the case of continuation of stationary solutions with continuous rotation symmetry.

The numerical methods we present have been implemented in the C code SYMPERCON [20]. In Section 5, examples are presented to illustrate the performance of the developed algorithmic tools. In Subsection 5.1 we use both SYMPERCON, AUTO [8] and CONTENT [17] for the computation of the period doubling cascade of the Lorenz equations to demonstrate the better performance of SYMPERCON. In Section 5.2 we compute symmetry breaking bifurcations of periodic orbits of four coupled cells. In Section 5.3 we show how oscillations of an electric circuit can be computed efficiently by exploiting their spatio-temporal symmetry.

We partly follow the unpublished manuscripts [20, 22, 23]. For a description of the program SYMPERCON see $[22,20]$.

\section{Acknowledgements}

This paper is dedicated to the memory of Karin Gatermann who died on New Year's Day 2005. She pioneered the use of symmetry methods in numerical bifurcation theory and introduced one of us (CW) to this topic back in 1991. Karin gave us lots of valuable advice and encouragement and was a dear friend.

CW was partially supported by the Nuffield Foundation and by a European Union Marie Curie fellowship under contract number HPMF-CT-2000-00542, AS acknowledges funding by a European Union Marie Curie studentship at the Marie-Curie Training Site "University of Warwick" during the academic year 2001/2002. CW thanks the Freie Universität Berlin, in particular the research group of Bernold Fiedler, for their hospitality during visits when parts of this paper were written. We both thank Mark Roberts for many helpful discussions and for inviting us to the Warwick Symposium on Geometric Mechanics and Symmetry in 2001/2002 during which this project was started. Finally we acknowledge the EU-TMR network MASIE for funding our participation in various summer schools, workshops and conferences.

\section{Continuation of symmetric periodic orbits}

In this section we review the multiple shooting method of Deuflhard [5] for the computation of periodic orbits, show how symmetries of periodic orbits can be exploited within the multiple shooting approach and present a continuation method for symmetric periodic orbits based on the Gauss-Newton method.

\subsection{Computation of single periodic orbits - single shooting method}

In this subsection we briefly recollect the algorithm for the computation of periodic orbits of an autonomous ordinary differential equation (ODE)

$$
\dot{x}=f(x), f: \mathbb{R}^{n} \rightarrow \mathbb{R}^{n},
$$

which has been introduced in [5].

Let $\Phi_{t}(\cdot)$ be the flow of (2.1) and let $x(t)=\Phi_{t}\left(x^{*}\right)$ be a periodic solution of (2.1) of period $T^{*}$, i.e., $x\left(T^{*}\right)=x^{*}$. Then any time shifted solution $x\left(t+t_{0}\right), t_{0} \in \mathbb{R}$, is also a periodic solution, because the system (2.1) is autonomous. All these solutions determine the same periodic orbit

$$
\mathcal{P}=\mathcal{P}_{x(\cdot)}=\{x(t), t \in \mathbb{R}\} .
$$


In order to avoid this non-uniqueness a well known analytical technique is to fix a Poincaré section $S=S_{x^{*}}$ which is an $(n-1)$-dimensional affine hyperplane transversal to the periodic orbit $\mathcal{P}$ at the point $x^{*}$, see e.g. [16]. Let us use the Poincaré section orthogonal to the orbit

$$
S=S_{x^{*}}=x^{*}+S_{x^{*}}^{\prime} \quad \text { where } \quad S_{x^{*}}^{\prime}=\operatorname{span}\left(f\left(x^{*}\right)\right)^{\perp} .
$$

Then $x^{*}$ is a fixed point of the Poincaré map (first return map) $\Pi: S \rightarrow S$.

Definition 2.1 We say that a periodic orbit $\mathcal{P}$ with period $T^{*}$ is non-degenerate if

$$
\left.\mathrm{D}_{x} \Pi(x)\right|_{x=x^{*}}-\text { id }
$$

is regular for $x^{*} \in \mathcal{P}$.

In this case $x^{*}$ is a locally unique fixed point of the Poincaré map $\Pi$ and a locally unique root of the equation

$$
\mathcal{F}(x):=\Pi(x)-x=0, \quad \text { where } \mathcal{F}: S \rightarrow S .
$$

Numerically one can either fix the phase by an additional phase condition, as described eg in $[1,16]$, or solve an underdetermined equation, as in [5]. We follow the latter approach and compute a point $x=x^{*}$ on the periodic solution together with its period $T=T^{*}$ by solving the underdetermined equation $F(y)=0$. Here $F: \mathbb{R}^{n} \times \mathbb{R} \rightarrow \mathbb{R}^{n}$ is given by

$$
F(x, T)=\Phi_{T}(x)-x=0, \quad \text { where } y=(x, T) .
$$

We solve (2.2) by an underdetermined Gauss-Newton method:

$$
\begin{aligned}
\Delta y^{k} & =-\mathrm{D} F\left(y^{k}\right)^{+} F\left(y^{k}\right), \\
y^{k+1} & =y^{k}+\Delta y^{k},
\end{aligned}
$$

where $\mathrm{D} F\left(y^{k}\right)^{+}$denotes the Moore-Penrose pseudo-inverse of $\mathrm{D} F\left(y^{k}\right)$. Remember that for $A \in \operatorname{Mat}(m, n), m \leq n, \operatorname{rank} A=m, x \in \mathbb{R}^{n}, b \in \mathbb{R}^{m}, x=A^{+} b$ is defined by

$$
A x=b, \quad x \perp \operatorname{ker}(A)
$$

where $\operatorname{ker}(A)$ is the kernel of $A$. So $x=A^{+} b$ is the smallest in norm solution of $A x=b$ and hence the Newton correction $\Delta y^{k}$ is the smallest solution of the underdetermined linear system in (2.3).

The Jacobian $\mathrm{D} F(x, T)$ of $(2.2)$ in the solution $\left(x^{*}, T^{*}\right)$ is given by

$$
\mathrm{D} F\left(x^{*}, T^{*}\right)=\left[-\mathrm{id}+\mathrm{D}_{x} \Phi_{T^{*}}\left(x^{*}\right), f\left(\Phi_{T^{*}}\left(x^{*}\right)\right)\right]=\left[-\mathrm{id}+\mathrm{D}_{x} \Phi_{T^{*}}\left(x^{*}\right), f\left(x^{*}\right)\right] .
$$

Therefore a kernel vector $t^{f}$ of $\mathrm{D} F\left(y^{*}\right)$ at the solution point $y^{*}=\left(x^{*}, T^{*}\right)$ is the tangent $t^{f}=\left(f\left(x^{*}\right), 0\right)$ to the trajectory.

Remark 2.2 This approach can be interpreted as computing periodic orbits in an adaptive Poincaré section, which is approximately orthogonal to the periodic orbit: Since for the kernel vectors $t^{k}=\left(t_{x}^{k}, t_{T}^{k}\right)$ of $\mathrm{D} F\left(y^{k}\right)$ we have $t^{k} \rightarrow t^{f}$ as $k \rightarrow \infty$, the Gauss-Newton iterate $x^{k+1}=$ $x^{k}+\Delta x^{k}$ lies in the adaptive Poincaré section $S_{x^{k}}=x^{k}+\operatorname{span}\left(t_{x}^{k}\right) \perp \approx x^{*}+\operatorname{span}\left(f\left(x^{*}\right)\right)^{\perp}$.

If $x^{*}$ lies on a non-degenerate periodic orbit, i.e., if $\mathrm{D}_{x} \Pi\left(x^{*}\right)-$ id is regular, then by $(2.4)$ the Jacobian $\mathrm{D} F\left(x^{*}, T^{*}\right)$ is regular. Since this condition does not depend on the chosen point $x^{*}$ on $\mathcal{P}$ we get the following convergence result:

Proposition 2.3 If the periodic orbit $\mathcal{P}$ through $x^{*}=\Phi_{T^{*}}\left(x^{*}\right)$ is non-degenerate then there is a tubular neighbourhood $U$ of the periodic orbit $\mathcal{P}$ where there is no other periodic solution with period near $T^{*}$ and which is such that the Gauss-Newton method (2.3) applied to (2.2) converges for initial data $\hat{x} \in U$ and $\hat{T} \approx T^{*}$.

Before we review the extension of this basic shooting method to the multiple shooting context we show how symmetries of periodic orbits can be exploited numerically. 


\subsection{Symmetries of periodic orbits and how to exploit them}

Let $\Gamma \subseteq \mathrm{GL}(n)$ be a finite group and let $f$ be $\Gamma$-equivariant [11], ie:

$$
f(\gamma x)=\gamma f(x) \quad \forall x \in \mathbb{R}^{n}, \gamma \in \Gamma .
$$

This condition on the vectorfield (2.1) implies that if $x(t)$ is a solution of the dynamical system (2.1) then also $\gamma x(t)$ is a solution. Hence the flow $\Phi_{t}(\cdot)$ of $(2.1)$ is also $\Gamma$-equivariant: $\gamma \Phi_{t}\left(x_{0}\right)=$ $\Phi_{t}\left(\gamma x_{0}\right)$ for every $\gamma \in \Gamma, x_{0} \in \mathbb{R}^{n}$.

For any $x \in \mathbb{R}^{n}$ the element $\gamma x$ is called conjugate to $x[11]$. An element $\gamma \in \Gamma$ is called a symmetry of $x \in \mathbb{R}^{n}$ if $\gamma x=x$; the set of all symmetries of $x$ (isotropy subgroup of $x$ ) is given by $K=\Gamma_{x}=\{\gamma \in \Gamma \mid \gamma x=x\}$. It can be seen easily that the vectorfield $f$ of (2.1) maps the fixed point space of $K$

$$
\operatorname{Fix}(K)=\left\{x \in \mathbb{R}^{n} \mid \gamma x=x \forall \gamma \in K\right\}
$$

into itself. Thus we can restrict the ODE (2.1) to the fixed point space $\operatorname{Fix}(K) \simeq \mathbb{R}^{n_{\text {red }}}$ which has a lower dimension $n_{\text {red }} \leq n$. In this way we obtain a symmetry reduced system $f_{\text {red }}: \mathbb{R}^{n_{\text {red }}} \rightarrow$ $\mathbb{R}^{n_{\text {red }}}$ which can be computed symbolically (see Gatermann, Hohmann [10]). The symmetry group acting on $\operatorname{Fix}(K)$ is $N(K) / K$ where $N(K)$ is the normalizer of $K$.

Remark 2.4 [11] If a finite group (or, more generally, a compact group) $\Gamma$ acts linearly on the phase space $X=\mathbb{R}^{n}$, i.e., if

$$
\gamma x:=\vartheta(\gamma) x, \quad \gamma \in \Gamma, x \in X,
$$

where $\vartheta: \Gamma \rightarrow \operatorname{GL}(n)$ is a homomorphism, then the phase space $X=\mathbb{R}^{n}$ can be decomposed into a sum $\mathbb{R}^{n}=X_{1} \oplus X_{2} \oplus \ldots \oplus X_{l}$ where the $X_{i}$ are $\Gamma$-invariant vector spaces and can not be decomposed into smaller $\Gamma$-invariant subspaces. Such vectorspaces $X_{i}$ are called $\Gamma$-irreducible and their corresponding reduced group actions $\vartheta_{i}:=\left.\vartheta\right|_{X_{i}}$ are called irreducible representations of the action of $\Gamma$ on $\mathbb{R}^{n}$. If a $\Gamma$-irreducible subspace $X$ is also irreducible as a vectorspace over $\mathbb{C}$, then its irreducible representation $\left.\vartheta\right|_{X}$ is called an absolutely irreducible representation. If it is reducible over $\mathbb{C}$ it is called a complex irreducible representation. We will encounter the concept of irreducible representations in the computation of bifurcations, c.f. Section 4.2.

The spatial symmetries $K$ of periodic solutions $x(t)$ are those group elements $\gamma \in \Gamma$ which leave each point on the periodic orbit invariant:

$$
K:=\Gamma_{x(t)}=\{\gamma \in \Gamma \mid \gamma x(t)=x(t) \forall t\}
$$

Since the flow $\Phi_{t}$ is $\Gamma$-equivariant the set of spatial symmetries $K$ of a periodic solution $x(t)$ does not depend on the time $t$. In addition to spatial symmetries there are also spatio-temporal symmetries which leave the periodic orbit $\mathcal{P}:=\mathcal{P}_{x(\cdot)}$ invariant as a whole but not pointwise, i.e., the spatio-temporal symmetries of a periodic orbit $\mathcal{P}$ are given by

$$
L:=\{\gamma \in \Gamma \mid \gamma \mathcal{P}=\mathcal{P}\}
$$

Each $\gamma \in L$ corresponds to a phase shift $\Theta(\gamma) T^{*}$ of the $T^{*}$-periodic solution $x(t)$ :

$$
\gamma \in L \Rightarrow x(t)=\gamma x\left(t+\Theta(\gamma) T^{*}\right), \text { where } \Theta(\gamma) \in S^{1} \simeq \mathbb{R} / \mathbb{Z}
$$

So spatio-temporal symmetries come in pairs $(\gamma, \Theta(\gamma)) \in \Gamma \times S^{1}$. We define an action of the spatio-temporal symmetry group $\Gamma \times S^{1}$, where $S^{1}=\mathbb{R} / \mathbb{Z}$, on $T^{*}$-periodic solutions $x(t)$ of $(2.1)$ as follows:

$$
((\gamma, \theta) x)(t):=\gamma x\left(t+\theta T^{*}\right), \quad(\gamma, \theta) \in \Gamma \times S^{1}
$$


Note that $\Theta: L \rightarrow \mathbb{R} / \mathbb{Z}$ is a group homomorphism with the spatial symmetries $K$ as kernel and that

$$
L / K \equiv \mathbb{Z}_{\ell}, \quad \ell \in \mathbb{N},
$$

see [11]. The spatial symmetries of periodic solutions can be exploited by restriction onto the fixed point space $\operatorname{Fix}(K)$, i.e., by using a symmetry reduced system $f_{\text {red }}: \operatorname{Fix}(K) \rightarrow \operatorname{Fix}(K)$.

From now on we assume that the spatial symmetry $K$ of the periodic orbit is trivial. Then the spatio-temporal symmetries of the periodic orbit form a finite cyclic group $L=\mathbb{Z}_{\ell}$. In bifurcation theory the spatio-temporal symmetries of periodic orbits are taken into account by studying the reduced Poincaré map. It was first introduced by Fiedler [9] and later used by Lamb, Melbourne and Wulff $[18,19]$ in order to classify symmetry breaking bifurcations of periodic orbits, see also Section 3. Let $\alpha \in L=\mathbb{Z}_{\ell}$ be that element in $L$ that corresponds to the smallest possible non-zero phase shift $T^{*} / \ell$ :

$$
\alpha x\left(t+\frac{T^{*}}{\ell}\right)=x(t) \quad \forall t .
$$

We call this spatio-temporal symmmetry the drift symmetry of the periodic orbit [25]. For $x^{*} \in \mathcal{P}$ define the Poincaré section as usual by $S=x^{*}+\operatorname{span}\left(f\left(x^{*}\right)\right)^{\perp}$. Then the reduced Poincaré map is defined as

$$
\Pi_{\mathrm{red}}=\alpha \hat{\Pi}, \quad \hat{\Pi}: S \rightarrow \alpha^{-1} S,
$$

where $\alpha$ is the drift symmetry of the periodic orbit, i.e., satisfies (2.9), and $\hat{\Pi}$ maps $x \in S$ into the point where the positive semi-flow through $x$ first hits $\alpha^{-1} S[9]$. The fixed point equation $\Pi_{\text {red }}(x)=x$ or, equivalently, the equation

$$
\mathcal{F}(x)=\Pi_{\text {red }}(x)-x=0, \quad \text { where } \mathcal{F}: S \rightarrow S,
$$

then determines periodic orbits with spatio-temporal symmetry $L$. Note that in the case of trivial symmetry $\ell=1, \alpha=\mathrm{id}$, the reduced Poincaré map $\Pi_{\text {red }}$ becomes the standard Poincaré map $\Pi$ introduced in Section 2.1. In order to numerically exploit spatio-temporal symmetries we proceed as in Section 2.1: Each point $x$ on a $T$-periodic orbit with drift symmetry $\alpha$ satisfies the underdetermined equation

$$
F: \mathbb{R}^{n} \times \mathbb{R} \rightarrow \mathbb{R}^{n}, \quad F(x, T)=\alpha \Phi_{\frac{T}{\ell}}(x)-x=0 .
$$

This system is analogous to the corresponding underdetermined system (2.2) in the case of trivial symmetry and reduces to it in the case $\alpha=\mathrm{id}, \ell=1$. It can also be solved by a Gauss-Newton method. Note that it suffices to compute the flow $\Phi_{t}(\cdot)$ and the Wronskian matrix $\mathrm{D}_{x} \Phi_{t}(\cdot)$ only up to time $\frac{T}{\ell}$ instead of $T$, which is a remarkable reduction of the computational cost in the case of high spatio-temporal symmetry. In a solution point $\left(x^{*}, T^{*}\right)$ we have

$$
\mathrm{D} F\left(x^{*}, T^{*}\right)=\left[\alpha \mathrm{D}_{x} \Phi_{\frac{T^{*}}{\ell}}\left(x^{*}\right)-\mathrm{id}, \frac{1}{\ell} f\left(\alpha \Phi_{\frac{T^{*}}{\ell}}\left(x^{*}\right)\right)\right]=\left[\alpha \mathrm{D}_{x} \Phi_{\frac{T^{*}}{\ell}}\left(x^{*}\right)-\mathrm{id}, \frac{1}{\ell} f\left(x^{*}\right)\right],
$$

analogous to the case of trivial symmetry, c.f. (2.4). In particular $\mathrm{D} F\left(x^{*}, T^{*}\right)$ is closely related to $\mathrm{D}_{x} \mathcal{F}\left(x^{*}\right)=\left.\mathrm{D}_{x} \Pi_{\text {red }}(x)\right|_{x=x^{*}}-$ id. We extend the definition of non-degeneracy to symmetric periodic orbits as follows:

Definition 2.5 We say that a symmetric periodic orbit $\mathcal{P}$ with drift symmetry $\alpha$ is non-degenerate if $\mathrm{D} \Pi_{\text {red }}\left(x^{*}\right)-\mathrm{id}$ is regular for $x^{*} \in \mathcal{P}$ where $\Pi_{\text {red }}$ is from (2.10).

From (2.12) we conclude that $\mathrm{D} F\left(x^{*}, T^{*}\right)$ in the periodic orbit is regular if and only if the periodic orbit is non-degenerate, and so we get the following proposition which is analogous to Proposition 2.3: 
Proposition 2.6 If the symmetric periodic orbit through $x^{*}=\alpha \Phi_{\frac{T^{*}}{\ell}}\left(x^{*}\right)$ is non-degenerate in the sense of Definition 2.5 then there is a tubular neighbourhood $U^{\ell}$ about the periodic orbit $\mathcal{P}$ through $x^{*}$ with the property that there is no other periodic orbit with symmetry $L$ and period near $T^{*}$ in $U$ and which is such that the Gauss-Newton method (2.3) applied to (2.11) converges for initial values $\hat{x} \in U, \hat{T} \approx T^{*}$.

\subsection{Multiple shooting approach}

In order to numerically compute unstable symmetric periodic solutions we use the just described algorithm in the multiple shooting context (c.f. [5]): we compute $k$ points on a periodic orbit with spatio-temporal symmetry $L=\mathbb{Z}_{\ell}$, trivial isotropy and drift symmetry $\alpha$ by solving the underdetermined equation

$$
F\left(x_{1}, \ldots, x_{k}, T\right)=0, \quad F: \mathbb{R}^{N} \rightarrow \mathbb{R}^{M},
$$

where $N=M+1=k n+1,0=s_{1}<\ldots<s_{k+1}=1$ is a partition of the unit interval, $\Delta s_{i}=s_{i+1}-s_{i}$ for $i=1, \ldots k$, and

$$
F_{i}\left(x_{1}, \ldots, x_{k}, T\right)= \begin{cases}\Phi_{\Delta s_{i} T}\left(x_{i}\right)-x_{i+1} & \text { for } i=1, \ldots, k-1, \\ \alpha \Phi_{\frac{\Delta s_{k} T}{\ell}}\left(x_{k}\right)-x_{1} & \text { for } i=k .\end{cases}
$$

The linear systems which arise in the Gauss-Newton method are of the form $J y=b$, where $y=(x, T) \in \mathbb{R}^{n k+1}, x=\left(x_{1}, \ldots, x_{k}\right), b=\left(b_{1}, \ldots, b_{k}\right)$,

$$
J=\mathrm{D} F(x, T)=\left(\begin{array}{cccccc}
G_{1} & -\mathrm{id} & & & & g_{1} \\
& G_{2} & -\mathrm{id} & & & g_{2} \\
& & \ddots & \ddots & & \vdots \\
& & & G_{k-1} & -\mathrm{id} & g_{k-1} \\
-\mathrm{id} & & & & G_{k} & g_{k}
\end{array}\right)=[G, g],
$$

where $G$ is an $(n k, n k)$-matrix, $g$ an $n k$-vector, and

$$
\begin{aligned}
& G_{i}=\mathrm{D}_{x} \Phi_{\frac{\Delta s_{i} T}{\ell}}\left(x_{i}\right), \quad i=1,2, \ldots, k-1, \\
& G_{k}=\alpha \mathrm{D}_{x} \Phi_{\frac{\Delta s_{k} T}{\ell}}\left(x_{k}\right), \\
& g_{i}=\mathrm{D}_{T} F_{i}(x, T)=\mathrm{D}_{T} \Phi_{\frac{\Delta s_{i} T}{\ell}}\left(x_{i}\right)=\frac{\Delta s_{i}}{\ell} f\left(\Phi_{\frac{\Delta s_{i} T}{\ell}}\left(x_{i}\right)\right), \quad i=1, \ldots, k-1, \\
& g_{k}=\frac{\Delta s_{k}}{\ell} \alpha f\left(\Phi_{\frac{\Delta s_{k} T}{\ell}}\left(x_{k}\right)\right) .
\end{aligned}
$$

We have

$$
J y=b \Leftrightarrow[G, g]\left(\begin{array}{l}
x \\
T
\end{array}\right)=b \Leftrightarrow G x=b-g T,
$$

so we can apply Gaussian block elimination to $G$ to solve these linear systems. This yields the following algorithm:

1.) Compute the condensed right hand side

$$
b_{c}:=C(G, b, k)=b_{k}+G_{k} b_{k-1}+\cdots+G_{k} \cdots G_{2} b_{1} .
$$

2.) Compute the condensed matrix $\left[G_{c}, g_{c}\right]$ with

$$
G_{c}:=G_{k} \cdots G_{1}, \quad g_{c}:=C(G, g, k) .
$$


3.) Compute a solution of the condensed system $\left[G_{c}-\mathrm{id}, g_{c}\right]\left(\begin{array}{c}x_{1} \\ T\end{array}\right)=b_{c}$, e.g.

$$
\left(\begin{array}{c}
x_{1} \\
T
\end{array}\right)=\left[G_{c}-\mathrm{id}, g_{c}\right]^{+} b_{c},
$$

using QR-decomposition.

4.) Compute $x$ via the explicit recursion

$$
x_{i}=G_{i-1} x_{i-1}-b_{i-1}+g_{i-1} T \text { for } \quad i=2, \ldots, k .
$$

We have now obtained a solution $y=J^{-} b$ where $y=(x, T)$ and $J^{-}$is an outer inverse of $J$. In order to compute the solution $J^{+} b$ where $J^{+}$is the Moore-Penrose pseudo-inverse of $J$ we have to add one more step:

5.) Compute the kernel vector $t=\left(t_{x}, t_{T}\right)$ of $J$, where $t_{x}=\left(t_{1}, t_{2}, \ldots, t_{k}\right)$. Starting from a tangent of the condensed system $\left(\begin{array}{l}t_{1} \\ t_{T}\end{array}\right)$

$$
\left[G_{c}-\mathrm{id}, g_{c}\right]\left(\begin{array}{c}
t_{1} \\
t_{T}
\end{array}\right)=0
$$

we obtain a tangent $t$ of the whole system by

$$
t_{i}=G_{i-1} t_{i-1}+g_{i-1} t_{T} \quad \text { for } \quad i=2, \ldots, k .
$$

In the end we project $y \rightarrow y-\frac{\langle y, t\rangle}{\langle t, t\rangle} t$.

An easy computation shows that in a solution point $y^{*}=\left(x^{*}, T^{*}\right)$ we have

$$
\left[G_{c}-\mathrm{id}, g_{c}\right]=\left[\alpha \mathrm{D}_{x} \Phi_{\frac{T^{*}}{\ell}}\left(x_{1}^{*}\right)-\mathrm{id}, \frac{1}{\ell} f\left(x_{1}^{*}\right)\right],
$$

so the condensed matrix $E_{c}:=\left[G_{c}-\mathrm{id}, g_{c}\right]$ equals the Jacobian (2.12) of the single shooting approach in the point $x_{1}^{*}$. The Jacobian $J$ is regular iff $\left[G_{c}-\mathrm{id}, g_{c}\right]$ is regular. Hence we get the following result which is analogous to Proposition 2.6:

Theorem 2.7 The Jacobian $J$ of the multiple shooting system (2.13) is regular at the symmetric periodic orbit if and only if the periodic orbit is non-degenerate in the sense of Definition 2.5. In this case the Gauss-Newton method (2.3) applied to (2.13) converges for sufficiently good initial data.

\section{Remarks 2.8}

a) The approach for symmetry exploitation in the multiple shooting approach can be transferred to collocation methods (used in AUTO and CONTENT $[8,17]$ ) since collocation can be viewed as a special case of a multiple shooting method where the number of grid points corresponds to the number of multiple shooting points $k$ and the initial value problem solver consists of only one integration step of a collocation method. The advantage of the multiple shooting approach is that it allows the use of adaptive order and stepsize initial value problem solvers for the computation of the flow $\Phi_{t}(x)$ and the Wronskians $\mathrm{D}_{x} \Phi_{t}(x)$ which we use for the evaluation of $F$ rsp. DF, like the extrapolation codes of Deufhard [6]. These techniques have been implemented in the code SYMPERCON [22, 20], see Section 5.1 for a comparison with AUTO and CONTENT. 
b) Note that in the program packages AUTO and CONTENT $[8,17]$ a phase condition is used to obtain a unique periodic orbit, whereas we solve an underdetermined equation for the periodic orbit. Since we use the Moore-Penrose pseudo-inverse to compute the corrections of the Gauss-Newton method $\Delta y^{k}=-\mathrm{D} F\left(y^{k}\right)^{+} F\left(y^{k}\right)$, we have $\Delta y^{k} \perp \operatorname{ker}\left(\mathrm{D} F\left(y^{k}\right)\right)$. Here

$$
\operatorname{ker}\left(\operatorname{D} F\left(y^{k}\right)\right) \approx \operatorname{ker}\left(\operatorname{D} F\left(y^{*}\right)\right)=\operatorname{span}\left(t^{f}\right),
$$

where

$$
t^{f}=\left(f\left(x_{1}^{*}\right), \ldots, f\left(x_{k}^{*}\right), 0,0\right) .
$$

The condition $\Delta y^{k} \perp \operatorname{ker}\left(\mathrm{D} F\left(y^{k}\right)\right)$ is therefore an "adaptive phase condition" which is such that the correction $\Delta y^{k}$ is the smallest solution of the equation for the Newton corrections $\mathrm{D} F\left(y^{k}\right) \Delta y^{k}=-F\left(y^{k}\right)$, c.f. Remark 2.2.

c) Dellnitz $[3,4]$ computes symmetric periodic orbits by a Galerkin ansatz based on Fourier modes. This method is effective near Hopf bifurcations since in this situation periodic orbits can be approximated by few Fourier modes.

\subsection{Continuation of nondegenerate periodic orbits}

In this section we show how the pathfollowing method for stationary solutions described in [7] can be extended to the case of symmetric periodic solutions. We consider the parameter dependent $\Gamma$-equivariant dynamical system $\dot{x}=f(x, \lambda)$ from (1.1) again and first look at stationary solutions of (1.1),

$$
f(y)=0, \quad f: \mathbb{R}^{n+1} \rightarrow \mathbb{R}^{n}, \quad y=(x, \lambda) .
$$

If $y^{*}=\left(x^{*}, \lambda^{*}\right)$ is a stationary solution and $\mathrm{D}_{y} f(y)$ is regular at $y^{*}$ then $(2.20)$ locally defines a solution branch. We apply the tangential continuation method based on implicit reparametrization presented in [7] to compute this solution branch. By writing $y=(x, \lambda)$ we want to express that the parameter $\lambda$ does not play any extraordinary role so that turning points can be treated easily. The pathfollowing algorithm works as follows: if a solution $y^{*}$ is given a new guess $\hat{y}$ is computed by setting $\hat{y}=y^{*}+\epsilon t\left(y^{*}\right)$ where $t(y)$ is the normalized kernel vector of $\mathrm{D}_{y} f(y)$, and hence the continuation tangent, and $\epsilon$ is a suitably chosen stepsize. Then an underdetermined Gauss-Newton method as in (2.3) is used for the iteration from the guess $\hat{y}$ back to the solution path. The stepsize control is described in [7]. We now show how to apply this continuation method to symmetric periodic orbits.

\subsubsection{Single shooting method}

In the case of symmetric periodic solutions we want to compute fixed points of the parameter dependent reduced Poincaré map $\Pi_{\text {red }}: S \times \mathbb{R} \rightarrow S$

$$
\Pi_{\text {red }}(x, \lambda)=x \quad \Leftrightarrow \quad \mathcal{F}(x, \lambda):=\Pi_{\text {red }}(x, \lambda)-x=0,
$$

or, equivalently, solutions of the parameter dependent nonlinear equation

$$
\mathcal{F}(x, \lambda)=0, \quad \mathcal{F}: S \times \mathbb{R} \rightarrow S .
$$

We can in principle apply the above described continuation method to this equation. The continuation tangent in a solution point $\left(x^{*}, \lambda^{*}\right)$ is simply the kernel vector $t_{\mathcal{F}}^{*}$ of $\mathrm{D} \mathcal{F}\left(x^{*}, \lambda^{*}\right)$. But in the numerical realization of this idea we want to use the method of adaptive Poincaré sections of Sections 2.1, 2.2, c.f. Remark 2.2. So we again introduce the period as a new variable and solve (in the single shooting approach) the underdetermined equation

$$
F: \mathbb{R}^{n+2} \rightarrow \mathbb{R}^{n}, \quad F(x, T, \lambda)=\alpha \Phi_{\frac{T}{\ell}}(x, \lambda)-x=0,
$$


by a Gauss-Newton procedure. Now the kernel $\operatorname{ker}(\mathrm{D} F)$ of $\mathrm{D} F$ is two-dimensional. In a solution point $\left(x^{*}, \lambda^{*}\right)$ one kernel vector of $\mathrm{D} F$ is the tangent to the periodic orbit

$$
t^{f}=\left(f\left(x^{*}, \lambda^{*}\right), 0,0\right) \in \operatorname{ker}\left(\mathrm{D} F\left(x^{*}, T^{*}, \lambda^{*}\right)\right) .
$$

We want to determine the continuation tangent $t^{*}$ in such a way that it corresponds to the theoretical tangent vector $t_{\mathcal{F}}^{*}$. First the continuation tangent has to be in the kernel of $\mathrm{D} F$, and second the continuation tangent should lie in the Poincaré section $S$. Since we choose the Poincaré section orthogonal to the orbit, this leads to the conditions

$$
t^{*} \in \operatorname{ker}(\mathrm{D} F), \quad t^{*} \perp t^{f} .
$$

The Jacobian in the solution point $\left(x^{*}, T^{*}, \lambda^{*}\right)$ is given by

$$
\mathrm{D} F\left(x^{*}, T^{*}, \lambda^{*}\right)=\left[\alpha \mathrm{D}_{x} \Phi_{\frac{T^{*}}{\ell}}\left(x^{*}, \lambda^{*}\right)-\mathrm{id}, \frac{1}{\ell} f\left(x^{*}, \lambda^{*}\right), \alpha \mathrm{D}_{\lambda} \Phi_{\frac{T^{*}}{\ell}}\left(x^{*}, \lambda^{*}\right)\right],
$$

and therefore we get the following proposition, analogous to Proposition 2.6:

Proposition 2.9 Let $x^{*}$ lie on a $T^{*}$-periodic orbit $\mathcal{P}$ of (1.1) with symmetry $L=\mathbb{Z}_{\ell}$ and drift symmetry $\alpha$. Then the Jacobian $\operatorname{DF}\left(x^{*}, T^{*}, \lambda^{*}\right)$ is regular if and only if

$$
\mathrm{D} \mathcal{F}\left(x^{*}, \lambda^{*}\right)=\left[\mathrm{D}_{x} \mathcal{F}\left(x^{*}, \lambda^{*}\right), \mathrm{D}_{\lambda} \mathcal{F}\left(x^{*}, \lambda^{*}\right)\right]=\left[\mathrm{D}_{x} \Pi_{\text {red }}\left(x^{*}\right)-\mathrm{id}, \mathrm{D}_{\lambda} \Pi_{\text {red }}\left(x^{*}\right)\right]
$$

is regular. In this case the path of periodic solutions given by (2.21) is locally unique in the following sense: there is a tubular neighbourhood $U$ of the periodic orbit $\mathcal{P}$ such that every periodic solution $\hat{x}(t)$ in this neighbourhood with period $\hat{T}$ close to $T^{*}$, parameter $\hat{\lambda}$ close to $\lambda^{*}$ and drift symmetry $\alpha$ lies on the path of periodic solutions defined by (2.21). Moreover the Gauss-Newton method (2.3) applied to (2.22) converges to a periodic solution on this path for initial data $\hat{x} \in U, \hat{T} \approx T^{*}, \hat{\lambda} \approx \lambda^{*}$.

Note that for non-degenerate periodic orbits the condition of Proposition 2.9 is always satisfied, but it also holds in a turning point bifurcation, see Section 2.5.

\subsubsection{Multiple shooting ansatz}

In the multiple shooting approach we solve the parameter dependent equation

$$
F\left(x_{1}, \ldots, x_{k}, T, \lambda\right)=0, \quad F: \mathbb{R}^{n k} \times \mathbb{R} \times \mathbb{R} \rightarrow \mathbb{R}^{n k},
$$

where $F$ is as in (2.14). Nearly everything carries over from Section 2.3, we just have one more column in the Jacobian consisting of the parameter derivatives

$$
P_{i}=\mathrm{D}_{\lambda} \Phi_{\frac{\Delta s_{i} T}{\ell}}\left(x_{i}, \lambda\right), \quad i=1, \ldots, k-1, \quad P_{k}=\alpha \mathrm{D}_{\lambda} \Phi_{\frac{\Delta_{k} T}{\ell}}\left(x_{k}, \lambda\right) .
$$

Therefore, to solve the linear equations $J y=b, y=\left(x_{1}, \ldots, x_{k}, T, \lambda\right)$, we have to compute an additional condensed vector (step 1 in the Gaussian block elimination algorithm), namely

$$
p_{c}:=C(P)=P_{k}+G_{k} P_{k-1}+\cdots+G_{k} \cdots G_{2} P_{1} .
$$

The condensed matrix in steps 2 and 3 is of the form $\left[G_{c}-\mathrm{id}, g_{c}, p_{c}\right]$, the recursion (step 4 ) has to be modified to

$$
x_{i}=G_{i-1} x_{i-1}-b_{i-1}+g_{i-1} T+P_{i-1} \lambda \quad \text { for } \quad i=2, \ldots, k,
$$


and in step 5 we compute an orthonormal basis of the 2-dimensional kernel of $J$ and project the preliminary solutions $y=J^{-} b$ onto the orthogonal complement of this kernel.

As can be seen from the Gaussian block elimination, $J$ has full rank if the condensed matrix $E_{c}:=\left[G_{c}-\mathrm{id}, g_{c}, p_{c}\right]$ has full rank. A simple computation shows that the matrix $E_{c}$ equals the Jacobian of the single shooting approach (2.24). Thus the Gauss-Newton method (2.3) applied to the multiple shooting system of equations (2.26) converges under the same conditions to a solution as the Gauss-Newton method of the single shooting method, namely if the assumptions of Proposition 2.9 are satisfied.

As in the case of the single shooting method, we choose the continuation tangent $t^{*}$ as the kernel vector of $\mathrm{D} F\left(y^{*}\right)$ (with $F$ from (2.26)) which is orthogonal to $t^{f}$ from (2.19).

\subsection{Turning points}

Before we come to the detection and computation of bifurcations of symmetric periodic orbits in Sections 3 and 4 we first consider turning points of symmetric periodic orbits. We saw that periodic orbits are solutions of the equation $\mathcal{F}(x, \lambda)=0$ where $\mathcal{F}$ is as in (2.21). Turning points are characterized by the condition that $\mathrm{D}_{x} \mathcal{F}\left(x^{*}, \lambda^{*}\right)$ from (2.25) is singular, but that $\mathrm{D} \mathcal{F}\left(x^{*}, \lambda^{*}\right)$ has full rank. In this case the solution path $(x(s), \lambda(s))$, of $(2.21), s \in \mathbb{R}, x(0)=x^{*}, \lambda(0)=\lambda^{*}$, satisfies $\lambda^{\prime}(0)=0$. Generically $\lambda^{\prime \prime}(0) \neq 0$ so that the solution path has a turning point in $\lambda$. Turning points can be detected by a change of sign of the $\lambda$-component $t_{\lambda}^{*}$ of the continuation tangent $t^{*}$ of the periodic solution provided this test is done after the tests of other bifurcations. This ordering of the monitoring tests for bifurcations is important, because, as we will see in Sections 3.2, 3.5 and 4, a change of sign of the $\lambda$-component $t_{\lambda}^{*}$ of the continuation tangent $t^{*}$ also occurs at flip up bifurcations and Hopf points along periodic orbits.

A turning point between two points $y^{(0)}=\left(x^{(0)}, T^{(0)}, \lambda^{(0)}\right)$ and $y^{(1)}=\left(x^{(1)}, T^{(1)}, \lambda^{(1)}\right)$ with continuation tangents $t^{(0)}, t^{(1)}$ respectively is detected if

$$
t_{\lambda}^{(0)} t_{\lambda}^{(1)}<0
$$

The $\lambda$-component of the turning point is then computed by Hermite interpolation exactly in the same way as in the case of stationary solutions, see [7]: We construct a cubic polynomial $y(\tau)=(x(\tau), T(\tau), \lambda(\tau))$, where $y:[0,1] \rightarrow \mathbb{R}^{N}$, over the line $y^{(0)}+\tau\left(y^{(1)}-y^{(0)}\right), \tau \in[0,1]$, such that

$$
\begin{aligned}
& y(0)=y^{(0)}, \quad y^{\prime}(0)=\frac{\left\|y^{(1)}-y^{(0)}\right\|^{2}}{\left\langle y^{(1)}-y^{(0)}, t^{(0)}\right\rangle} t^{(0)}, \\
& y(1)=y^{(1)}, \quad y^{\prime}(1)=\frac{\left\|y^{(1)}-y^{(0)}\right\|^{2}}{\left\langle y^{(1)}-y^{(0)}, t^{(1)}\right\rangle} t^{(1)},
\end{aligned}
$$

and solve the quadratic equation $\frac{\mathrm{d} \lambda}{\mathrm{d} \tau}(\tau)=0$ for its unique root $\hat{\tau} \in[0,1]$. We then take the value $\hat{y}=y(\hat{\tau})$ of the Hermite polynomial at $\hat{\tau}$ as initial guess for a Gauss-Newton iteration. The periodic solution $y^{*}$ obtained in this way is accepted as turning point if

$$
\left|t_{\lambda}^{*}\right|<\text { tol }
$$

where $t^{*}$ is the continuation tangent at $y^{*}$ and tol is the required accuracy of the computation. Otherwise we replace $y^{(0)}$ or $y^{(1)}$ by $y^{*}$ so that $t_{\lambda}^{(0)} t_{\lambda}^{(1)}<0$ and repeat the procedure.

\section{Computation of flip down and flip up bifurcations}

In this section we show how generic bifurcations of symmetric periodic orbits to other periodic orbits can be computed numerically. This involves the detection and numerical computation of bifurcation points and the computation of the start off directions for the bifurcating branches. 
We only have to follow non-conjugate branches and distinguish between two types of symmetry changing bifurcations: there are symmetry increasing bifurcations which lead to a super group of the symmetry group of the original solution, i.e., the bifurcating solutions possess more symmetry, and we have symmetry breaking bifurcations which lead to a subgroup of the symmetry group of the original solution.

In this section we only treat bifurcations from periodic orbits to periodic orbits, not Hopf bifurcations (from periodic orbits to stationary solutions) - we will treat these in the next section. Such bifurcations have been classified by Fiedler [9] in the case of cyclic symmetry groups. Bifurcations of periodic orbits in systems with arbitrary finite symmetry group were classified by Lamb and Melbourne [18], see also [19].

In this paper we assume that the symmetry group of the dynamical system is discrete and that the isotropy $K$ of the periodic orbit is trivial, i.e., we restrict the dynamical system to the corresponding fixed point space $\operatorname{Fix}(K)$. In other words, we do not treat bifurcations to periodic orbits with smaller or bigger isotropy group $K$. The methods we present extend the techniques of Gatermann and Hohmann [10] for the numerical computation of symmetry changing bifurcations of stationary solutions to the case of periodic solutions. In particular, the symmetry monitoring functions which are used for the detection of symmetry changing bifurcations are related to those used in [10].

Generic bifurcations of periodic orbits with trivial isotropy to other periodic orbits are caused by a period doubling (flip down) or period halving (flip up) bifurcation of the reduced Poincaré map $[18,19]$. We start with generic bifurcations without symmetry where the ODE (1.1) is not assumed to be equivariant.

\subsection{Detection and computation of period doubling bifurcations}

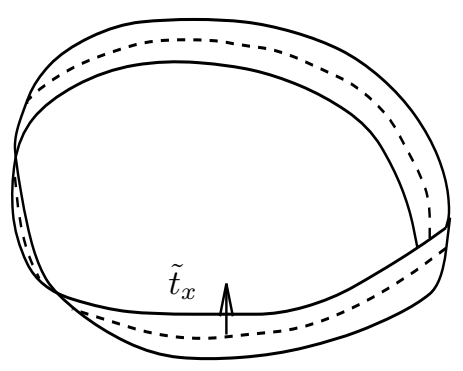

Figure 1: Period doubling bifurcation in phase space. The bifurcating periodic orbit lies on a Mobius strip. Dashed line: original periodic orbit, solid line: bifurcating periodic orbit with twice the period, $\tilde{t}_{x}: x$-component of the continuation tangent for the bifurcating branch.

A point $\left(x^{*}, \lambda^{*}\right)$ characterizing a periodic solution with period $T^{*}$ is a period doubling bifurcation point (flip down point) if the Jacobian $\mathrm{D}_{x} \Pi$ of the Poincaré map has a single eigenvalue -1 in $\left(x^{*}, \lambda^{*}\right)$ with eigenvector $v_{S}^{*} \in S$ and if this is the only eigenvalue on the complex unit circle, see e.g. $[13,16]$. Let $x(\lambda)$ be the solution branch of $\Pi(x, \lambda)=x$ with $x\left(\lambda^{*}\right)=x^{*}$. Then we moreover require that the path $\mu(\lambda)$ of eigenvalues of $\mathrm{D}_{x} \Pi(x(\lambda), \lambda)$ with $\mu\left(\lambda^{*}\right)=-1$ satisfies the generic transversality condition

$$
\frac{\partial \mu}{\partial \lambda}\left(\lambda^{*}\right) \neq 0 .
$$


Under these assumptions $\left(x^{*}, \lambda^{*}\right)$ is a pitchfork bifurcation point of the map

$$
\tilde{\mathcal{F}}(x, \lambda)=\Pi(\Pi(x, \lambda), \lambda)-x=0,
$$

see $[13,16]$. The normal form of this bifurcation is

$$
\tilde{f}(z, \lambda)=z^{3}-\lambda z .
$$

By a Lyapounov-Schmidt reduction (3.2) can be reduced to a scalar equation in $z=\left\langle x-x^{*}, v_{S}^{*}\right\rangle$. After a suitable change of coordinates this scalar equation takes the form (3.3), up to order 3 , see $[13,16]$. The vector $\tilde{t}_{\tilde{\mathcal{F}}}=\left(v_{S}^{*}, 0\right)$ is the tangent vector of the bifurcating branch in $\left(x^{*}, \lambda^{*}\right)$. The bifurcating periodic orbits correspond to fixed points of $\Pi^{2}$ and hence have approximately twice the period of the original periodic solution. They lie on a Mobius strip around the original periodic orbit, see Figure 1 . The map $\tilde{\mathcal{F}}$ is $\mathbb{Z}_{2}$-equivariant where the nonlinear $\mathbb{Z}_{2}$-action is given by the Poincaré map $(x, \lambda) \rightarrow(\Pi(x), \lambda)$ (in the normal form (3.3) this $\mathbb{Z}_{2}$-symmetry becomes $z \rightarrow-z$ ). So a period doubling bifurcation is a $\mathbb{Z}_{2}$-symmetry breaking bifurcation of equilibria of $\tilde{\mathcal{F}}$. If we consider the $T$-periodic solutions on the original branch as $\widetilde{T}$-periodic, where $\widetilde{T}:=2 T$, the original branch has temporal $\mathbb{Z}_{2}$-symmetry for the action of the spatio-temporal symmetry group $\Gamma \times \mathbb{R}$ given by $(2.7)$ :

$$
(\text { id, } \theta) x(t)=x(t+\theta \widetilde{T})=x(t) \quad \text { for } \quad \theta=1 / 2,
$$

and the branching solutions are not $\mathbb{Z}_{2}$-symmetric. So we see that even in the case of a trivial symmetry group $\Gamma=\{\mathrm{id}\}$ the period doubling bifurcation corresponds to a $\mathbb{Z}_{2}$-symmetry breaking bifurcation as the phase shift symmetry (temporal symmetry) of the periodic orbit is broken.

In the following we briefly describe how to numerically detect and compute period doubling bifurcations in non-symmetric systems. We adapt standard techniques used in the context of collocation methods $[1,8,17]$ to our approach for the computation of periodic orbits as solutions of underdetermined systems, as described in Section 2. In Section 3.5 we show how to adapt these methods to the numerical computation of symmetry breaking bifurcations of symmetric periodic orbits.

\subsubsection{Numerical detection of period doubling bifurcations}

Period doublings can be detected by a change of sign of

$$
d(\lambda):=\operatorname{det}\left(\mathrm{D}_{x} \Phi_{T^{*}}\left(x^{*}, \lambda^{*}\right)+\mathrm{id}\right)
$$

which occurs due to the transversality condition (3.1). The matrix $\mathrm{D}_{x} \Phi_{T^{*}}\left(x^{*}, \lambda^{*}\right)$ is computed in the single shooting approach to obtain $\mathrm{D} F\left(x^{*}, T^{*}, \lambda^{*}\right)$, see (2.4), and also in the multiple shooting approach in the computation of the condensed matrix $G_{c}$, see (2.18) with $\ell=1$ and $\alpha=$ id.

\subsubsection{Computation of period doubling bifurcation points}

If a period doubling point has been detected it can be computed by use of linear interpolation and a Gauss-Newton procedure to iterate back to the solution path: If there is a period doubling point between two consecutively computed periodic solutions $y^{(0)}$ and $y^{(1)}$ then

$$
d\left(\lambda^{(0)}\right) d\left(\lambda^{(1)}\right)<0 .
$$


By linear interpolation of the two points $\left(\lambda^{(0)}, d\left(\lambda^{(0)}\right)\right)$ and $\left(\lambda^{(1)}, d\left(\lambda^{(1)}\right)\right)$ we obtain a point $(\hat{\lambda}, 0)$ which gives us an approximation for the parameter value $\hat{\lambda}$ of the bifurcation point. Linear interpolation between the points $y^{(0)}$ and $y^{(1)}$ provides a first guess $\hat{y}$ with parameter $\hat{\lambda}$ for the period doubling point. This guess is then iterated back to a point $y^{*}$ on the solution path by a Gauss-Newton procedure. If

$$
\left\|y^{*}-\hat{y}\right\| \leq \text { tol, }
$$

$y^{*}$ is accepted as period doubling point. If not, then either $y^{(0)}$ or $y^{(1)}$ is replaced by $y^{*}$, such that the condition $d\left(\lambda^{(0)}\right) d\left(\lambda^{(1)}\right)<0$ is satisfied, and the procedure is repeated.

Doubling the number of multiple shooting points for the bifurcating branch If we fix the number of multiple shooting points on the bifurcating branch of $2 T$-periodic solutions, then after some period doubling bifurcations the multiple shooting method is likely to diverge because the initial number of multiple shooting points will not be appropriate for a periodic solution with a much higher period than the original period. Therefore it is preferable to compute the bifurcating branch of periodic orbits with twice as many multiple shooting points, i.e., to set $\tilde{k}=2 k$ as the number of multiple shooting points of the bifurcating branch. The bifurcation point $\tilde{y}=\left(\tilde{x}_{1}, \ldots, \tilde{x}_{\tilde{k}}, \tilde{T}, \tilde{\lambda}\right)$ on the bifurcating branch is given by

$$
\tilde{x}_{i}=x_{i}^{*}, \quad \tilde{x}_{i+k}=x_{i}^{*} \quad \text { for } \quad i=1, \ldots, k, \quad \tilde{T}=2 T^{*}, \quad \tilde{\lambda}=\lambda^{*},
$$

and the multiple shooting nodes $\tilde{s}_{i}, i=0,1, \ldots, \tilde{k}$, of the bifurcating branch are set to

$$
\tilde{s}_{i}=\frac{s_{i}}{2}, \quad \tilde{s}_{i+k}=\frac{1+s_{i}}{2}, \quad i=1, \ldots, k .
$$

\subsubsection{Computation of start off directions for the bifurcating branch}

After a period doubling bifurcation point has been found the start off direction for the bifurcating branch has to be computed. The continuation tangent of the original periodic branch is just the usual continuation tangent. The start off direction for the bifurcating branch is computed as follows:

Single shooting ansatz We first consider the single shooting approach: we want the start off direction for the bifurcating branch of periodic orbits to be orthogonal to the $T^{*}$-periodic orbit, so that it lies in the Poincare section $S$. In $S \times \mathbb{R}$ the tangent of the bifurcating branch in the bifurcation point should be the vector $\tilde{t}_{\tilde{\mathcal{F}}}=\left(v_{S}^{*}, 0\right)$ where $v_{S}^{*}$ is the eigenvector of $\mathrm{D}_{x} \Pi\left(x^{*}\right)$ to the eigenvalue -1 , see above. It can be computed by projecting the kernel vector $v^{*}$ of $\mathrm{D}_{x} \Phi_{T}\left(x^{*}, \lambda^{*}\right)+$ id onto the orthogonal complement of the tangent $f\left(x^{*}\right)$ to the periodic orbit through $x^{*}$ : Let

$$
\tilde{t}_{x}=v^{*}-\frac{\left\langle v^{*}, f\left(x^{*}\right)\right\rangle}{\left\langle f\left(x^{*}\right), f\left(x^{*}\right)\right\rangle} f\left(x^{*}\right) .
$$

Then we take

$$
\tilde{t}=\left(\tilde{t}_{x}, \tilde{t}_{T}, \tilde{t}_{\lambda}\right)=\left(\tilde{t}_{x}, 0,0\right)
$$

as the start off direction for the bifurcating periodic solutions. In phase space the bifurcating periodic solutions lie on a Moebius band in the middle of which is the original $T^{*}$-periodic solution. The start off direction is tangential to the Moebius band and orthogonal to the original solution (see Figure 1). 
Multiple shooting ansatz As preliminary tangent vector $v_{1}^{*}$ of the bifurcating branch for the first multiple shooting point we choose the eigenvector of $G_{c}+\mathrm{id}$ to -1 where $G_{c} \approx \mathrm{D}_{x} \Phi_{T^{*}}\left(x^{*}\right)$ is the condensed matrix, see $(2.16,2.18)$. As preliminary tangent start off directions at the multiple shooting points $x_{2}^{*}, \ldots, x_{k}^{*}$ we take

$$
v_{j}^{*}=G_{j} v_{j-1}^{*}, \quad j=2, \ldots, k .
$$

The first $n k$ components of the start off tangent $\tilde{t}$ of the bifurcating branch in the multiple shooting approach are then obtained by projecting $v^{*}=\left(v_{1}^{*}, \ldots, v_{k}^{*}\right)$ to the orthogonal complement of the vector $t^{f}=\left(f\left(x_{1}^{*}\right), \ldots, f\left(x_{k}^{*}\right)\right)$.

Since the number of multiple shooting points is doubled on the bifurcating branch (see Section 3.1 .2 ), this gives us only the first half of the $x$-component

$$
\tilde{t}_{x}=\left(\tilde{t}_{1}, \tilde{t}_{2}, \ldots, \tilde{t}_{k}, \tilde{t}_{k+1}, \ldots, \tilde{t}_{2 k}\right)
$$

of the start off tangent $\tilde{t}=\left(\tilde{t}_{x}, 0,0\right)$ of the bifurcating branch. The whole $x$-component of $\tilde{t}$ is obtained by copying the first half into the second half and multiplying it with -1 , i.e.,

$$
\tilde{t}_{k+i}=-\tilde{t}_{i}, \quad i=1, \ldots k .
$$

As in the single shooting approach we have $\tilde{t}_{T}=\tilde{t}_{\lambda}=0$.

\subsection{Detection and computation of period halving bifurcations}

In this section we describe an algorithm for the detection and computation of period halving bifurcations (flip up points) along branches of periodic orbits. Again, we assume that the symmetry group $\Gamma$ of the dynamical system (1.1) is trivial.

\subsubsection{Detection of period halving bifurcations}

Period halvings can be detected as follows: for a solution point $y=\left(x_{1}, \ldots, x_{k}, T, \lambda\right)$ of $(2.26)$ we compute

$$
u(y):=\Phi_{\frac{T}{2}}\left(x_{1}, \lambda\right)-x_{1}=x_{j}-x_{1} .
$$

Here one multiple shooting node $s_{j}$ is set to $s_{j}=1 / 2$ so that no additional initial value problem has to be solved.

If there is a period halving point $y^{*}=\left(x^{*}, T^{*}, \lambda^{*}\right)$ between two consecutively computed periodic solutions $y^{(0)}$ and $y^{(1)}$ the vector $u$ goes through zero. As we saw before, a flip bifurcation corresponds to a pitchfork bifurcation of (3.2). Figure 2 shows the normal form (3.3) of the pitchfork bifurcation and points $x^{(0)}$ and $x^{(1)}$ corresponding to periodic orbits before and after passing the bifurcation. For each parameter value $\lambda$, the corresponding point on the solid and dashed curve belong to the same periodic orbit, and are conjugate points with respect to the $\mathbb{Z}_{2}$-symmetry action which, for $(3.2)$, is given by $x \rightarrow \Pi(x, \lambda)$, see above. For the points $x^{(0)}$ and $x^{(1)}$ we denote the difference vectors by $u^{(\nu)}=\Pi\left(x^{(\nu)}, \lambda^{(\nu)}\right)-x^{(\nu)}, \nu=0,1$. From Figure 2 we see that the vectors $u^{(0)}$ and $u^{(1)}$ are approximately parallel with opposite sign. At the numerically computed solutions $y^{(0)}$ and $y^{(1)}$ the vectors $u\left(y^{(0)}\right)$ and $u\left(y^{(1)}\right)$ are good approximations for $u^{(0)}$ and $u^{(1)}$. Therefore a period halving point can be detected by the following condition on the angle between $u\left(y^{(0)}\right)$ and $u\left(y^{(1)}\right)$ :

$$
\frac{\left\langle u\left(y^{(0)}\right), u\left(y^{(1)}\right)\right\rangle}{\left\|u\left(y^{(0)}\right)\right\|\left\|u\left(y^{(1)}\right)\right\|}<0 .
$$



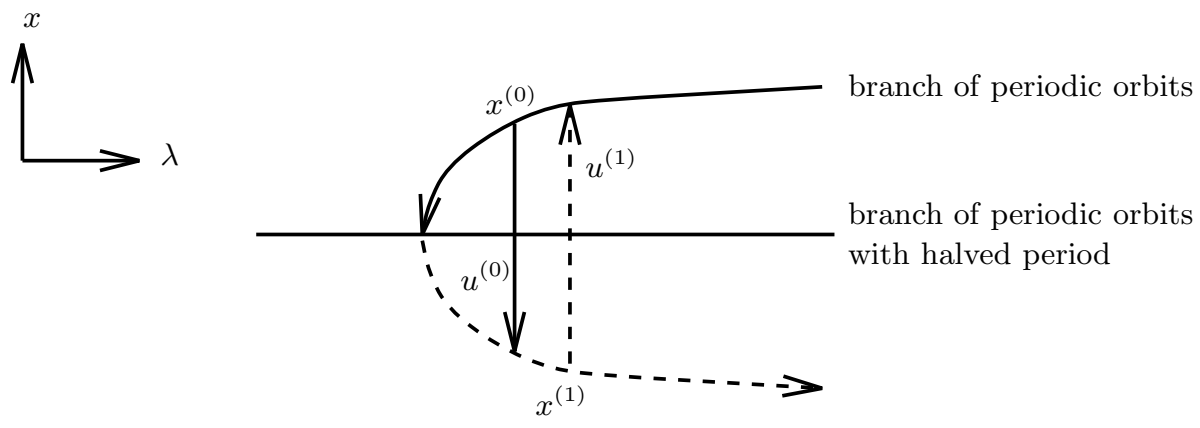

Figure 2: Detection of period halvings using (3.6). Solid curve: branch of periodic orbits before passing the flip up point; dashed curve: branch of periodic orbits after passing the flip up point; See text for more explanations.

\subsubsection{Computation of period halving bifurcation points and start off directions}

Assume that a period halving bifurcation has been detected between two consecutively computed periodic solutions $y^{(0)}$ and $y^{(1)}$. We now describe a method for the computation of period halving points which is based on existing methods for detecting period doubling points. The algorithm consists of the following steps:

1. We obtain a first guess for the location of the flip up point by approximating the solution branch by a Hermite interpolating polynomial, c.f. Figure 2. We compute a Hermite polynomial $y(\tau)$ of degree 3 through the two points $y(0)=y^{(0)}, y(1)=y^{(1)}$ of the bifurcation diagram in between of which a period halving has been detected. This is analogous to the Hermite interpolation used to locate turning points, see Section 2.5. By computing the extremum $\lambda(\hat{\tau})$ of the polynomial $\lambda(\tau)$ where $y(\tau)=(x(\tau), T(\tau), \lambda(\tau))$ we obtain a first guess $\hat{y}=y(\hat{\tau})=\left(\hat{x}_{1}, \ldots, \hat{x}_{k}, \hat{T}, \hat{\lambda}\right)$ for the flip up point. We now halve the period $\hat{T}:=\hat{T} / 2$. Again, as in the case of period doubling bifurcations, the number of multiple shooting points is adapted with respect to changes of the period. In this case the number of multiple shooting points is $\tilde{k}=j$ where $j$ is such that $s_{j}=\frac{1}{2}$. Decreasing the number of multiple shooting points at flip up bifurcations obviously speeds up the calculation time.

2. Now we use this first guess as starting value for a flip down point computation. We look for a flip down point in the parameter scope $\left[\lambda_{\min }, \lambda_{\max }\right]$ where

$$
\lambda_{\min }=\hat{\lambda}-\epsilon, \quad \lambda_{\max }=\hat{\lambda}+\epsilon, \quad \epsilon=\max \left(\operatorname{tol}, \min \left(\left|\lambda^{(0)}-\hat{\lambda}\right|,\left|\lambda^{(1)}-\hat{\lambda}\right|\right)\right),
$$

and tol is the prescribed accuracy. The period doubling bifurcation point on this branch is then accepted as period halving point for the original branch.

\section{Remarks 3.1}

a) In AUTO and CONTENT period halving bifurcations are not properly detected: the programs detect bifurcation points of "unknown type", but does not compute the bifurcating branch of periodic solutions with halved period.

b) Note that period halving bifurcations can not be detected by a change of sign of a determinant, in contrast to transcritical and saddle node bifurcations of equilibria. The monitoring function that we use is inspired by the monitoring functions for symmetry increasing bifurcations of equilibria developed by Gatermann and Hohmann [10]. 
c) In the computation of bifurcations of equilibria numerical methods based on extended systems are frequently employed (see e.g. $[1,16]$ ). One could of course also use such a method to locate period halving bifurcations. A period halving point could for example be computed by solving the system

$$
0=F(x, T, \lambda, v):=\left(\begin{array}{l}
\Phi_{T / 2}(x ; \lambda)-x \\
\mathrm{D}_{x} \Phi_{T / 2}(x ; \lambda) v+v \\
\|v\|^{2}-1
\end{array}\right)
$$

using a Newton type method. But since this requires the approximation of the second derivative of the flow map it would be more expensive than the method that we suggest.

\subsection{Bifurcations of periodic orbits with $\mathbb{Z}_{p}$-symmetry}

In this section we deal with generic symmetry changing but isotropy preserving secondary bifurcations of symmetric periodic orbits. The right hand side $f$ of the ODE (1.1) is assumed to be $\Gamma$-equivariant under a finite group $\Gamma \subset \mathrm{GL}(n)$, as in Section 2.2. We assume that the spatial symmetry $K$ of the periodic orbit is trivial (or restrict the dynamics to Fix $(K)$ ). This implies that the spatio-temporal symmetry of the periodic orbits is cyclic: $L \simeq \mathbb{Z}_{\ell}$. We can then, without loss of generality, restrict to the case $\Gamma \simeq \mathbb{Z}_{p}$ for $p$ a multiple of $\ell$, see [18]. We describe how the generic secondary bifurcations of periodic orbits with $\mathbb{Z}_{p}$-symmetry, which have been classified by Fiedler [9], can be treated numerically. In this section we only deal with bifurcations of periodic orbits into other periodic orbits (not Hopf bifurcations along branches of periodic orbits).

So let $x^{*}$ lie on a periodic orbit $\mathcal{P}$ with period $T^{*}$, trivial isotropy $K$, spatio-temporal symmetry $L=\mathbb{Z}_{\ell}$ and drift symmetry $\alpha$. Define the Poincaré section as usual by $S=x^{*}+$ $\operatorname{span}\left(f\left(x^{*}\right)\right)^{\perp}$. To examine bifurcations of symmetric periodic orbits the reduced Poincaré map

$$
\Pi_{\mathrm{red}}=\alpha \hat{\Pi}, \quad \hat{\Pi}: S \rightarrow \alpha^{-1} S,
$$

from (2.10) is used where $\hat{\Pi}$ maps points of $S$ into the points where the positive semi-flow through $x$ first hits $\alpha^{-1} S$. In the case of trivial isotropy $K$ the relationship between the full Poincaré map $\Pi$ and the reduced Poincaré map $\Pi_{\text {red }}$ is given by

$$
\Pi=\alpha^{-\ell} \Pi_{\text {red }}^{\ell}=\Pi_{\text {red }}^{\ell} .
$$

Here we used that $\alpha^{\ell}=$ id. Generic bifurcations of symmetric periodic orbits are bifurcations of the reduced Poincaré map $\Pi_{\text {red }}$ which arise from an eigenvalue \pm 1 of the Jacobian $\mathrm{D}_{x} \Pi_{\text {red }}$, see $[9,18]$.

Generic bifurcations of $\Pi_{\text {red }}$ without breaking of the spatial symmetry are turning points and period doublings/halvings (flip down and flip up bifurcations); turning points of $\Pi_{\text {red }}$ lead to turning points of the full Poincaré map $\Pi$. They can be detected and computed in the same way as in the case of no symmetry, see Section 2.5.

Flip down bifurcations of the reduced Poincaré map $\Pi_{\text {red }}$ lead to pitchfork bifurcations respectively period doubling bifurcations of $\Pi$ depending on whether $\ell$ is odd or even, see [9]. If $\ell$ is odd, in particular if the symmetry is trivial (i.e. $\ell=1$ ), we have a flip doubling (period doubling) bifurcation. If $\ell$ is even then a flip pitchfork bifurcation takes place where the period is preserved, but the spatio-temporal symmetry halved.

\subsubsection{Flip pitchfork bifurcation}

First we consider the flip pitchfork bifurcation. Let $\ell$ be even. Assume that the reduced Poincaré map $\Pi_{\text {red }}$ undergoes a flip down bifurcation. Then the bifurcating solutions $\tilde{x}(s), s \in \mathbb{R}, \tilde{x}(0)=$ 
$x^{*}$, are fixed points of $\Pi_{\mathrm{red}}^{2}$. By (3.7),

$$
\Pi(\tilde{x}(s))=\left(\Pi_{\text {red }}^{2}\right)^{\ell / 2}(\tilde{x}(s))=\tilde{x}(s),
$$

and so the Poincaré map $\Pi$ undergoes a pitchfork bifurcation. Hence the branching solutions have approximately the same period but their spatio-temporal symmetry $\tilde{L}=\mathbb{Z}_{\ell / 2}, \tilde{\ell}=\ell / 2$ has been halved. The drift symmetry of the bifurcating periodic orbits is $\tilde{\alpha}=\alpha^{2}$.

\subsubsection{Flip doubling bifurcation}

Next we consider the flip doubling bifurcation, ie, let $\ell$ be odd. Since $\Pi_{\text {red }}^{2}(\tilde{x}(s))=\tilde{x}(s)$ and $\ell$ is odd the following can be concluded:

$$
\Pi^{2}(\tilde{x}(s))=\Pi_{\text {red }}^{2 \ell}(\tilde{x}(s))=\tilde{x}(s) \text { and } \Pi(\tilde{x}(s))=\Pi_{\text {red }}^{\ell}(\tilde{x}(s))=\Pi_{\text {red }}(\tilde{x}(s)) \neq \tilde{x}(s) .
$$

So the Poincaré map $\Pi$ undergoes a period doubling bifurcation without breaking the symmetry $\mathbb{Z}_{\ell}$ : the spatio-temporal symmetry group of the bifurcating branch is $\tilde{L}=\mathbb{Z}_{\tilde{\ell}}$ where $\tilde{\ell}=\ell$ and is generated by the order $\ell$ element $\tilde{\alpha}=\alpha$. Note that the flip doubling bifurcation reduces to the period doubling bifurcation of non-symmetric systems, see Section 3.1, with $\ell=1, \alpha=$ id whereas the flip pitchfork bifurcation does not occur for non-symmetric systems.

\subsection{Numerical computation of symmetry breaking bifurcations}

Since the generic bifurcations of periodic orbits with underlying symmetry group $\mathbb{Z}_{p}$ described above are generated by periodic doubling bifurcations of the reduced Poincaré map they can be treated numerically with the methods for the period doubling bifurcation described in Section 3.1 .

\subsubsection{Detection and computation of flip down bifurcations}

Flip down bifurcations are detected by the a sign change of

$$
d(\lambda)=\operatorname{det}\left(\alpha \mathrm{D}_{x} \Phi_{\frac{T^{*}}{\ell}}\left(x^{*}\right)+\mathrm{id}\right) \text {, where } \alpha \mathrm{D}_{x} \Phi_{\frac{T^{*}}{\ell}}\left(x^{*}\right)=G_{c},
$$

analogously to the case of no symmetry, see Section 3.1.1. Once a flip down bifurcation has been detected it can be computed analogously as in the case of non-symmetric systems, see Section 3.1 .2 .

\subsubsection{Initialization of the bifurcating branch}

Once a flip down point $\left(x^{*}, T^{*}, \lambda^{*}\right)$ on the original branch has been found, the starting point $\tilde{y}=(\tilde{x}, \tilde{T}, \tilde{\lambda})$ for the bifurcating branch has to be computed. We set $\tilde{T}=T^{*}$ for a flip pitchfork bifurcation and $\tilde{T}=2 T^{*}$ otherwise, and $\tilde{\lambda}=\lambda^{*}$. Since the number of multiple shooting points is doubled the second half of $\tilde{x}=\left(\tilde{x}_{1}, \ldots, \tilde{x}_{2 k}\right)$ will be computed by applying the symmetry matrix to the first points:

$$
\begin{aligned}
\tilde{x}_{i} & =x_{i}^{*} \text { for } i=1, \ldots, k, \\
\tilde{x}_{i+k} & =\alpha^{-1} x_{i}^{*} \text { for } i=1, \ldots, k .
\end{aligned}
$$

The tangent vector $\tilde{t}=\left(\tilde{t}_{x}, 0,0\right)$ of the bifurcating branch is computed in a similar way: The first $n k$ components $\left(\tilde{t}_{1}, \ldots, \tilde{t}_{k}\right)$ of the $x$-component $\tilde{t}_{x}$ of the tangent $\tilde{t}$ of the bifurcating branch are computed as in Section 3.1.3, with $\mathrm{D}_{x} \Phi_{T^{*}}\left(x^{*}\right)$ replaced by $\alpha \mathrm{D}_{x} \Phi_{\frac{T^{*}}{\ell}}\left(x^{*}\right)$. Then the second half of $\tilde{t}_{x}=\left(\tilde{t}_{1}, \ldots, \tilde{t}_{2 k}\right)$ is computed by applying the symmetry matrix to the first half and multiplying it with -1 :

$$
\tilde{t}_{i+k}=-\alpha^{-1} \tilde{t}_{i} \quad \text { for } \quad i=1, \ldots, k .
$$




\subsection{Numerical computation of symmetry increasing bifurcations}

In this section we extend the algorithms for the detection and computation of period halving points for non-symmetric systems (Section 3.2) to systems with symmetry. The main issue here is the identification of the possible spatio-temporal symmetries of the bifurcating solutions which are needed for both, the detection of bifurcations and the computation of the bifurcating branch.

\subsubsection{Detection of flip up bifurcations}

Similarly as in the non-symmetric case, see Section 3.2, flip up points on a branch of periodic orbits with spatio-temporal symmetry $L=\mathbb{Z}_{\ell}$, trivial isotropy and drift symmetry $\alpha$ are detected by the angle condition (3.6)

$$
\frac{\left\langle u\left(y^{(0)}\right), u\left(y^{(1)}\right)\right\rangle}{\left\|u\left(y^{(1)}\right)\right\|\left\|u\left(y^{(1)}\right)\right\|}<0
$$

where $y^{(0)}$ and $y^{(1)}$ are two consecutive points on a branch of periodic solutions and

$$
u(x):=\tilde{\alpha} \Phi_{\frac{T}{2 \ell}}\left(x_{1}, \lambda\right)-x_{1}=\tilde{\alpha} x_{j}-x_{1} .
$$

Here the multiple shooting node $s_{j}$ is set to $s_{j}=\frac{1}{2}$ and $\tilde{\alpha}$ is a group element $\tilde{\alpha}$ which satisfies

$$
\tilde{\alpha}^{2}=\alpha .
$$

We now need to classify the possible choices of $\tilde{\alpha}$ and decide whether a flip up doubling or a flip up pitchfork bifurcation occurs.

Theorem 3.2 Let $i$ be such that $\alpha=\gamma_{p}^{i}$ where $\gamma_{p}$ generates the symmetry group $\Gamma=\mathbb{Z}_{p}$ of (1.1). Similarly, write $\tilde{\alpha}=\gamma_{p}^{\tilde{i}}$. Then we have:

a) Either $\tilde{i}=\frac{i}{2}$ or $\tilde{i}=\frac{i+p}{2}$. Both these values for $\tilde{i}$ are possible if $p$ and $i$ are even, $\tilde{i}=\frac{i}{2}$ is a possible solution for $p$ odd, $i$ even, and $\tilde{i}=\frac{i+p}{2}$ for $p$ and $i$ odd.

b) (i) If

$$
\ell \tilde{i}=0 \bmod p
$$

then a flip up doubling bifurcation takes place. The order $\tilde{\ell}$ of the drift symmetry $\tilde{\alpha}$ of the bifurcating branch and its period $\tilde{T}$ in the bifurcation point then satisfy

$$
\tilde{\ell}=\ell, \quad \tilde{T}=\frac{T^{*}}{2}
$$

where $T^{*}$ is the period of the original periodic orbit at the bifurcation point.

(ii) If

$$
\tilde{\imath} \neq 0 \bmod p
$$

then a flip up pitchfork bifurcation takes place. The order $\tilde{\ell}$ of the drift symmetry $\tilde{\alpha}$ of the bifurcating branch and its period $\tilde{T}$ in the bifurcation point then satisfy

$$
\tilde{\ell}=2 \ell, \quad \tilde{T}=T^{*} .
$$

Proof. From (3.9) we get

$$
2 \tilde{i}=i \quad \bmod p
$$

and so

$$
\tilde{i}=(i+j p) / 2, \quad j \in \mathbb{N} .
$$


Possible solutions to (3.10) which are different modulo $p$ are

$$
\tilde{i}=i / 2, \quad \tilde{i}=(i+p) / 2 .
$$

This proves part a) of the theorem. The rest follows from the definitions of flip pitchfork and flip doubling bifurcations, see Sections 3.3.1 and 3.3.2.

Remark 3.3 Consider a periodic orbit with trivial spatio-temporal symmetry, i.e., $\alpha=$ id, $i=0$. Then, if the order $p$ of the symmetry group $\Gamma=\mathbb{Z}_{p}$ is odd and the $\Gamma$-action is free, every flip up bifurcation is a period halving bifurcation. If $p$ is even then a flip up bifurcation of $\Pi_{\text {red }}$ can be a period halving bifurcation or a flip up pitchfork bifurcation with $\tilde{\alpha}=\gamma_{p}^{\frac{p}{2}}$.

\subsubsection{Computation of flip up points and start off tangents}

Once the spatio-temporal symmetry of the bifurcating periodic orbit is identified the flip up point and the start off directions for the bifurcating branch can be computed in the same way as for non-symmetric systems, see Section 3.2.

\section{Computation of equivariant Hopf points}

In this section we show how equivariant Hopf points along branches of periodic orbits can be detected and computed. For sake of simplicity, we first consider the case of a trivial symmetry group $\Gamma=\{\operatorname{id}\}$.

\subsection{Hopf bifurcations for non-symmetric systems}

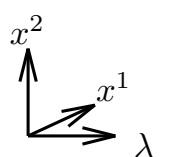

$$
\begin{gathered}
f^{(0)}=f\left(x^{(0)}\right) \\
f^{(1)}=f\left(x^{(1)}\right)
\end{gathered}
$$

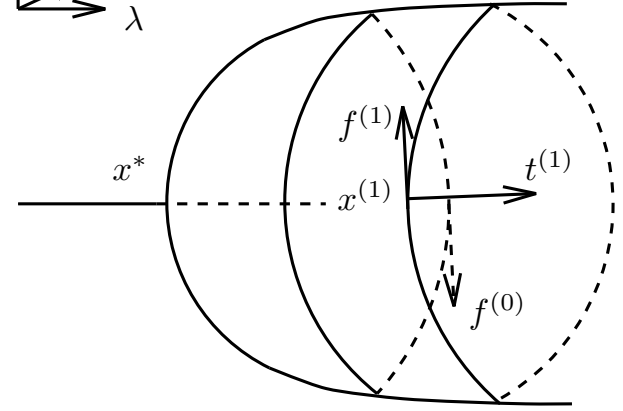

Figure 3: Detection of Hopf points between two periodic orbits $y^{(0)}=\left(x^{(0)}, T^{(0)}, \lambda^{(0)}\right)$ and $y^{(1)}=\left(x^{(1)}, T^{(1)}, \lambda^{(1)}\right)$ (single shooting method). Here $t^{(1)}$ is the continuation tangent at the point $y^{(1)}$.

A Hopf bifurcation point is a stationary solution $\left(x^{*}, \lambda^{*}\right)$, for which the Jacobian $\mathrm{D}_{x} f\left(x^{*}, \lambda^{*}\right)$ has a pair of purely imaginary eigenvalues $\pm \omega^{*} \mathrm{i}, \omega^{*} \neq 0$. We make the generic assumptions that these eigenvalues are simple, and that there is no resonance, i.e., no multiple $i \omega^{*} j, j \in \mathbb{N}_{0} \backslash\{1\}$, is an eigenvalue of $\mathrm{D}_{x} f\left(x^{*}, \lambda^{*}\right)$. Denote by $x(\lambda)$ the path of equilibria of (1.1) with parameter 
$\lambda$, so that in particular $x\left(\lambda^{*}\right)=x^{*}$. Let $\mu(\lambda)$ be the path of eigenvalues of $\mathrm{D}_{x} f(x(\lambda), \lambda)$ such that $\mu\left(\lambda^{*}\right)=\mathrm{i} \omega^{*}$ and assume that the generic transversality condition

$$
\operatorname{Re}\left(\frac{\partial \mu}{\partial \lambda}\left(\lambda^{*}\right)\right) \neq 0
$$

is satisfied. Then (see e.g. $[13,16])$ a unique branch $x(t ; \epsilon)$ of periodic solutions emanates from the stationary solution with small amplitude $O(\epsilon)$ and period $T(\epsilon) \approx T(0)=2 \pi / \omega^{*}$. This surface of periodic solutions is tangential to the real eigenspace $N_{\omega^{*}}$ of $\pm \omega^{*} \mathrm{i}$, ie $\mathrm{D}_{\epsilon} x(t ; 0) \in N_{\omega^{*}}$, and generically agrees (after a smooth coordinate change) to second order with a paraboloid $\lambda-\lambda^{*}=C\left(z_{1}^{2}+z_{2}^{2}\right)$, see e.g. $[13,16]$. We can consider the Hopf point $\left(x^{*}, \lambda^{*}\right)$ as an $S^{1}$-invariant $2 \pi / \omega^{*}$-periodic solution with respect to the action (2.7) of the temporal symmetry group on the periodic solutions $x(t)$ of $(1.1)$ :

$$
x^{*}(t) \equiv x^{*} \quad \forall t \Longrightarrow(\mathrm{id}, \theta) x^{*}(t)=x^{*}(t) \quad \forall t .
$$

Hence the Hopf bifurcation is an $S^{1}$-symmetry breaking bifurcation.

\subsubsection{Detection of Hopf points along branches of periodic orbits}

If a pathfollowing algorithm for periodic solutions runs through a Hopf point the continuation direction changes its sign and the same path of periodic orbits is computed again. Therefore Hopf points which occur during the pathfollowing of periodic orbits should be detected so that the pathfollowing routine can be stopped at the Hopf point. If there is a Hopf point between two consecutively computed periodic orbits $y^{(0)}$ and $y^{(1)}$, where $y=(x, T, \lambda), x=\left(x_{1}, \ldots, x_{k}\right)$, then the vectors $f\left(x_{i}\right)$, which are the infinitesimal generators of the $S^{1}$-symmetry in the point $x_{i}$, go through zero. Thus $f\left(x_{i}\right)$ is a symmetry monitoring function in this case. If the angle between the vectors $f\left(x_{i}\right)$ of two consecutively computed periodic orbits is greater than 90 degree, i.e., if for some $i \in\{1, \ldots, k\}$,

$$
\frac{\left\langle f\left(x_{i}^{(0)}\right), f\left(x_{i}^{(1)}\right)\right\rangle}{\left\|f\left(x_{i}^{(0)}\right)\right\|\left\|f\left(x_{i}^{(1)}\right)\right\|}<0
$$

then there is a Hopf point on the branch of periodic orbits between the $y^{(0)}$ and $y^{(1)}$, c.f. Figure 3.

Remark 4.1 Note that in the program packages AUTO [8] and CONTENT [17] the numerical part of which is based on AUTO Hopf points along periodic orbits are not detected properly. They are detected as general cycle branching points, but when switching to the bifurcating branch of stationary solutions an error occurs.

\subsubsection{Computation of Hopf bifurcations of non-symmetric systems}

If a Hopf point along a path of periodic orbits is detected, it can be computed by an extended system [12, 14], see also the review in [1]. We use a slightly different form of extended system which is underdetermined and does not fix the phase of the Hopf eigenvector to be computed. We present this extended system briefly in this subsection.

Let $x^{*}$ be Hopf point, i.e., an equilibrium $f\left(x^{*}, \lambda^{*}\right)=0$, the Jacobian $\mathrm{D}_{x} f\left(x^{*}, \lambda^{*}\right)$ of which has a pair of purely imaginary eigenvalues $\pm \mathrm{i} \omega^{*}$. Hence

$$
\mathrm{D}_{x} f\left(x^{*}, \lambda^{*}\right)\left(v^{*}+\mathrm{i} w^{*}\right)=\mathrm{i} \omega^{*}\left(v^{*}+\mathrm{i} w^{*}\right) .
$$

So we get

$$
\mathrm{D}_{x} f\left(x^{*}, \lambda^{*}\right) v^{*}=-\omega^{*} w^{*}, \quad \mathrm{D}_{x} f\left(x^{*}, \lambda^{*}\right) w^{*}=\omega^{*} v^{*}, \quad\left\|w^{*}\right\|^{2}+\left\|v^{*}\right\|^{2}=1 .
$$


Define $F: \mathbb{R}^{3 n+2} \rightarrow \mathbb{R}^{3 n+1}$ where

$$
F(x, \lambda, v, w, \omega)=\left(\begin{array}{c}
f(x, \lambda) \\
\mathrm{D}_{x} f(x, \lambda) v+\omega w \\
\mathrm{D}_{x} f(x, \lambda) w-\omega v \\
\langle v, v\rangle+\langle w, w\rangle-1
\end{array}\right) .
$$

Then $F=0$ yields the Hopf point and its imaginary eigenvalue and corresponding eigenvector. Moreover we have:

Theorem 4.2 If the eigenvalue $\pm \mathrm{i} \omega^{*}$ is simple, if $\mathrm{D}_{x} f\left(x^{*}, \lambda^{*}\right)$ is invertible and if the transversality condition (4.1) holds, then the Gauss-Newton method applied to (4.2) converges for sufficiently good initial data.

The proof of this theorem is basically contained in $[1,12,14]$. As shown there the transversality condition $\operatorname{Re} \mu^{\prime}\left(\lambda^{*}\right) \neq 0$ implies that any kernel vector $t=\left(t_{x}, t_{\lambda}, t_{v}, t_{w}, t_{\omega}\right)$ of the derivative $\mathrm{D} F\left(x^{*}, \lambda^{*}, v^{*}, w^{*}, \omega^{*}\right)$ of $F$ in the Hopf point satisfies $t_{\omega}=0, t_{\lambda}=0$ and $t_{x}=0$ and hence $\left(t_{v}, t_{w}\right)$ satisfies the equations

$$
\begin{aligned}
& 0=\mathrm{D}_{x} f\left(x^{*}, \lambda^{*}\right) t_{v}+\omega^{*} t_{w} \\
& 0=\mathrm{D}_{x} f\left(x^{*}, \lambda^{*}\right) t_{w}-\omega^{*} t_{v} \\
& 0=2\left\langle v^{*}, t_{v}\right\rangle+2\left\langle w^{*}, t_{w}\right\rangle .
\end{aligned}
$$

Therefore $t_{v}+\mathrm{i} t_{w}$ is a Hopf eigenvector. Equation (4.5) and the fact that the Hopf eigenvalue $\mathrm{i} \omega^{*}$ is a simple eigenvalue of $\mathrm{D}_{x} f\left(x^{*}, \lambda^{*}\right)$ imply that the kernel of $\mathrm{D} F$ in the Hopf point is onedimensional. Hence DF has full rank in the Hopf point and the Gauss-Newton method applied to (4.2) converges for sufficiently good initial data. As we will see later (see Sections 4.2 .1 and 5.2 ), symmetry often inforces multiple Hopf eigenvalues, so that the extended system (4.2) has to be modified in the case of equivariant Hopf points.

Initial guess for the Gauss-Newton iteration An initial guess for a Hopf point detected between two periodic orbits $y^{(0)}=\left(x^{(0)}, T^{(0)}, \lambda^{(0)}\right)$ and $y^{(1)}=\left(x^{(1)}, T^{(1)}, \lambda^{(1)}\right)$, where $x=$ $\left(x_{1}, \ldots, x_{k}\right)$, can be obtained by Hermite interpolation $y(\tau)$ between those points over the line $y^{(0)}+\tau\left(y^{(1)}-y^{(0)}\right), \tau \in[0,1]$, such that $y(0)=y^{(0)}, y(1)=y^{(1)}$, and by computing the point $\hat{y}=y(\hat{\tau})=(x(\hat{\tau}), T(\hat{\tau}), \lambda(\hat{\tau}))$ such that $\lambda^{\prime}(\hat{\tau})=0$, analogously to the computation of initial guesses for a turning point, see Section 2.5. We then set

$$
\hat{x}:=x_{1}(\hat{\tau}),
$$

define an approximation for the Hopf frequency $\hat{\omega}$ as

$$
\hat{\omega}=\frac{2 \pi}{\hat{T}}, \quad \text { where } \hat{T}=T(\hat{\tau}),
$$

and an approximation for the parameter value of the Hopf point as

$$
\hat{\lambda}=\lambda(\hat{\tau}) \text {. }
$$

We moreover define initial guesses $\hat{v}+\mathrm{i} \hat{w}$ for the Hopf eigenvector as

$$
\hat{v}=c \frac{\mathrm{d}}{\mathrm{d} \tau} x_{1}(\hat{\tau}), \quad \hat{w}=-\frac{1}{\hat{\omega}} \mathrm{D}_{x} f(\hat{x}, \hat{\lambda}) \hat{v}
$$

with $c$ such that

$$
\|\hat{v}\|^{2}+\|\hat{w}\|^{2}=1
$$

The point $(\hat{x}, \hat{\lambda}, \hat{v}, \hat{w}, \hat{\omega})$ is then used as initial guess for the Newton iteration applied to (4.2). 


\subsection{Detection and computation of equivariant Hopf points}

In this section we extend the methods for the computation of Hopf points of non-symmetric systems from Section 4.1 to systems with symmetry. The main issue here is how to deal with multiple Hopf eigenvalues forced by symmetry which lead to convergence failure of the extended system (4.2) for the computation of Hopf points of non-symmetric systems.

\subsubsection{Equivariant Hopf points}

We are starting from a $\Gamma$-invariant stationary solution $\left(x^{*}, \lambda^{*}\right)$, i.e., $\Gamma_{x^{*}}=\Gamma$. We assume that the Jacobian $\mathrm{D}_{x} f\left(x^{*}, \lambda^{*}\right)$ has a pair $\pm \omega^{*} \mathrm{i}$ of purely imaginary eigenvalues, $\omega^{*} \neq 0$, and that there are no resonances, i.e., $\pm j \mathrm{i} \omega^{*}, j=0,2,3,4, \ldots$, is not an eigenvalue of $\mathrm{D}_{x} f\left({ }^{*}, \lambda^{*}\right)$. As before let $N_{\omega^{*}}$ be the real eigenspace of $\mathrm{D}_{x} f\left(x^{*}, \lambda^{*}\right)$.

Lemma 4.3 [11] If $\gamma \in \Gamma_{x}$ then $\gamma \mathrm{D}_{x} f(x, \lambda)=\mathrm{D}_{x} f(x, \lambda) \gamma$. Moreover, every eigenspace of $\mathrm{D}_{x} f(x, \lambda)$ is $\Gamma_{x}$-invariant.

Proof. The first statement follows from the $\Gamma$-equivariance of $f$ and the $\gamma$-invariance of $x$. For the second statement let $u$ be a complex eigenvector of $A=\mathrm{D}_{x} f(x, \lambda)$ to the eigenvalue $\mu$. Since $\gamma A=A \gamma$ we have $A \gamma u=\gamma A u=\gamma \mu u$ so that $\gamma u$ is an eigenvector of $A$ to the eigenvalue $\mu$ as well.

As a consequence, $\mathrm{D}_{x} f\left(x^{*}, \lambda^{*}\right)$ is $\Gamma$-equivariant and $N_{\omega^{*}}$ is $\Gamma$-invariant. Hence $N_{\omega^{*}}$ can be decomposed into irreducible $\Gamma$-invariant subspaces, see Remark 2.4.

Definition 4.4 [11] We call an eigenvalue $\mu$ of a $\Gamma$-equivariant matrix $A$ a $\Gamma$-simple eigenvalue of $A$ if the real eigenspace $N$ of $A$ to the eigenvalue $\mu$ is irreducible.

We make the generic assumption that $\mathrm{i} \omega^{*}$ is a $\Gamma$-simple eigenvalue of $\mathrm{D}_{x} f\left(x^{*}, \lambda^{*}\right)$. This means that $\mathrm{i} \omega^{*}$ has the lowest multiplicity allowed by the symmetry group $\Gamma$.

Since $\mathrm{D}_{x} f\left(x^{*}, \lambda^{*}\right)$ is invertible, by the implicit function theorem applied to $\operatorname{Fix}(\Gamma)=\mathbb{R}^{n_{\text {red }}}$ there is a path $x(\lambda)$ of $\Gamma$-invariant equilibria of (1.1) with $x\left(\lambda^{*}\right)=x^{*}$. As in the case of the standard Hopf bifurcation we assume that the transversality condition (4.1) holds for the path $\mu(\lambda)$ of the pair of eigenvalues of $\mathrm{D}_{x} f(x(\lambda), \lambda)$ with $\mu\left(\lambda^{*}\right)=\mathrm{i} \omega^{*}$.

We define the operation of $\Gamma \times S^{1}$ on a $T$-periodic solution $x(t)$ as in $(2.7)$

$$
(\gamma, \theta) x(t)=\gamma x(t+\theta T) \text { for } \quad(\gamma, \theta) \in \Gamma \times S^{1},
$$

and the operation of $\Gamma \times S^{1}$ on the real eigenspace $N_{\omega^{*}}$ of $\pm \omega^{*}$ i of $\mathrm{D}_{x} f\left(x^{*}, \lambda^{*}\right)$ by

$$
(\gamma, \theta) u=\gamma e^{\theta \mathrm{D}_{x} f\left(x^{*}, \lambda^{*}\right) T^{*}} u, \quad(\gamma, \theta) \in \Gamma \times S^{1}, \quad u \in N_{\omega^{*}},
$$

where $T^{*}=\frac{2 \pi}{\omega^{*}}$.

Theorem 4.5 [11] (Equivariant Hopf Theorem) Let the above conditions be satisfied. If then for a subgroup $L \subset \Gamma \times S^{1}$ the fixed point space

$$
N_{\omega^{*}}^{L}:=\left\{u \in N_{\omega^{*}}:(\gamma, \theta) u=u \forall(\gamma, \theta) \in L\right\}
$$

satisfies the condition

$$
\operatorname{dim} N_{\omega^{*}}^{L}=2,
$$

then there is a unique branch $x(t ; \epsilon)$ of periodic solutions with amplitude $O(\epsilon)$ bifurcating from $\left(x^{*}, \lambda^{*}\right)$ with $\mathrm{D}_{\epsilon} x(t ; 0) \in N_{\omega^{*}}^{L}$, with parameter $\lambda(\epsilon)$, such that $\lambda(0)=\lambda^{*}$, with minimal periods $T(\epsilon)$, such that $T(0)=2 \pi / \omega^{*}$, and with $L$ as spatio-temporal symmetry group. 
As in the non-symmetric case the bifurcating periodic orbits generically lie on a paraboloid, see Figure 3.

Remark 4.6 The equivariant Hopf theorem provides the spatio-temporal symmetries $L$ of the bifurcating periodic orbits and the planes $N_{\omega^{*}}^{L}$ from which the start off directions for the emanating periodic orbits can be chosen: We define the phase space for the bifurcating periodic orbit with symmetry $L$ as $\operatorname{Fix}(K)$ where $K$, the group of spatial symmetries of the periodic orbit, is the kernel of the homomorphism $\Theta$ of the periodic orbit, see (2.6), and thus

$$
K=\{\gamma \in \Gamma, \quad(\gamma, 0) \in L\} .
$$

We compute the integer $\ell$ such that $L / K \simeq \mathbb{Z}_{\ell}$, see (2.8), and the drift symmetry $\alpha$ of the periodic orbit by determining the element $\left(\alpha, \theta^{*}\right)$ in $L$ with the smallest non-zero phase shift $\theta^{*}=\frac{1}{\ell}$, c.f. (2.9). We identify the Hopf point $\left(x^{*}, \lambda^{*}\right)$ with a periodic orbit which has period $T^{*}=\frac{2 \pi}{\omega^{*}}$, multiple shooting points $x_{i}^{*}=x^{*}$ for $i=1, \ldots k$ and the parameter $\lambda^{*}$. Similarly as in the non-symmetric case (c.f. [1]) we then define the continuation tangent $t^{*}=\left(t_{x}^{*}, t_{T}^{*}, t_{\lambda}^{*}\right)$ at this Hopf periodic orbit as follows:

$$
t_{T}^{*}=0, \quad t_{\lambda}^{*}=0, \quad t_{x}^{*}=\left(t_{1}^{*}, \ldots, t_{k}^{*}\right),
$$

where

$$
t_{i}^{*}=\cos \left(s_{i} T^{*} / \ell\right) v^{*}+\sin \left(s_{i} T^{*} / \ell\right) w^{*}, \quad i=1, \ldots, k .
$$

Here $v^{*}+\mathrm{i} w^{*}$ is the eigenvector to the purely imaginary eigenvalue $\omega^{*} \mathrm{i}$ of $\mathrm{D}_{x} f\left(x^{*}, \lambda^{*}\right)$ which is determined by the condition $v^{*}, w^{*} \in N_{\omega^{*}}^{L}$. See Sections 5.2 and 5.3 for applications.

\subsubsection{Detection of equivariant Hopf points}

Equivariant Hopf points along branches of periodic orbits are detected in the same way as Hopf points of non-symmetric systems, see Section 4.1.1.

\subsubsection{Computation of equivariant Hopf bifurcations}

As mentioned in Section 4.1.2 the extended system (4.2) for the computation of Hopf points of non-symmetric systems only converges if the Hopf eigenvalue $i \omega^{*}$ is simple. In the case of symmetric dynamical systems this assumption can only be satisfied if the corresponding irreducible representation is one-dimensional. In general the symmetry might enforce multiple eigenvalues (see Section 5.2 for an example). Therefore the numerical method for computing Hopf points from Section 4.1.2 has to be modified in the case of symmetric dynamical systems. In this section we present an efficient algorithm for computing equivariant Hopf points which applies to Hopf points which satisfy the conditions of the equivariant Hopf theorem.

Assume that an equivariant Hopf point $\left(x^{*}, \lambda^{*}\right)$ with Hopf eigenvalue $\pm \mathrm{i} \omega^{*}, \omega^{*}>0$, has been detected numerically along a branch of periodic orbits of the $\Gamma$-equivariant ODE (1.1) with drift symmetry $\alpha$ of order $\ell$ and, for simplicity, trivial isotropy $K$ (restrict the dynamics to $\operatorname{Fix}(K)$ and replace $\Gamma$ by $N(K) / K$ otherwise). As before we denote by $L \simeq \mathbb{Z}_{\ell}$ the spatio-temporal symmetry of the branch of periodic orbits. Then the Hopf point $x^{*}$ is $L$-invariant: $L \subseteq \Gamma_{x^{*}}$. We make the assumptions of the equivariant Hopf Theorem 4.5 replacing $\Gamma$ by $\Gamma_{x^{*}}$ and denote the plane tangent to the branch of periodic orbits at the Hopf point by $N_{\omega^{*}}^{L}$, as in (4.7).

We will now formulate an algorithm for the computation of the equivariant Hopf point $\left(x^{*}, \lambda^{*}\right)$ along with the Hopf frequency $\omega^{*}$ and the corresponding Hopf eigenvector $v^{*}+\mathrm{i} w^{*}$ satisfying $v^{*}, w^{*} \in N_{\omega^{*}}^{L}$. 
Note that the condition of the equivariant Hopf Theorem 4.5 that $N_{\omega^{*}}^{L}$ is 2-dimensional is equivalent to requiring that there is only one eigenvector $v^{*}+\mathrm{i} w^{*}$ of $\mathrm{D}_{x} f\left(x^{*}, \lambda^{*}\right)$ to the eigenvalue $\pm \mathrm{i} \omega^{*}$ satisfying

$$
v^{*}+\mathrm{i} w^{*}=\alpha \mathrm{e}^{\frac{2 \pi}{\omega^{*} \ell} \mathrm{D}_{x} f\left(x^{*}, \lambda^{*}\right)}\left(v^{*}+\mathrm{i} w^{*}\right) .
$$

Solving the nonlinear equation (4.9) numerically using an extended system would involve the computation of the exponential $\exp \left(\frac{2 \pi}{\omega \ell} \mathrm{D}_{x} f\left(x^{*}, \lambda^{*}\right)\right)$ of $\mathrm{D}_{x} f\left(x^{*}, \lambda^{*}\right)$ which is in general expensive. An extended system involving $\mathrm{D}_{x} f\left(x^{*}, \lambda^{*}\right)$ rather than its exponential, like the system (4.2) in the case of non-symmetric systems, is therefore preferable. To derive such a system we use the following approach: Note that

$$
\mathrm{D}_{x} f\left(x^{*}, \lambda^{*}\right)\left(v^{*}+\mathrm{i} w^{*}\right)=\mathrm{i} \omega^{*}\left(v^{*}+\mathrm{i} w^{*}\right)
$$

and so

and hence

$$
\exp \left(\frac{2 \pi}{\omega^{*} \ell} \mathrm{D}_{x} f\left(x^{*}, \lambda^{*}\right)\right)\left(v^{*}+\mathrm{i} w^{*}\right)=\mathrm{e}^{\frac{2 \pi \mathrm{i}}{\ell}}\left(v^{*}+\mathrm{i} w^{*}\right)
$$

$$
\alpha\left(v^{*}+\mathrm{i} w^{*}\right)=\mathrm{e}^{-\frac{2 \pi \mathrm{i}}{\ell}}\left(v^{*}+\mathrm{i} w^{*}\right) .
$$

So $v^{*}+\mathrm{i} w^{*}$ lies in the the complex eigenspace of $\alpha$ to the eigenvalue $\mathrm{e}^{-2 \pi \mathrm{i} / \ell}$ which we denote by $X_{\ell}^{c} \subset X^{c}=\mathbb{C}^{n}$, and $v^{*}$ and $w^{*}$ lie in the real eigenspace of $\alpha$ to the eigenvalue $e^{-2 \pi \mathrm{i} / \ell}$ which we denote by $X_{\ell}$. So $X_{\ell}$ is the $L$-invariant subspace of $X=\mathbb{R}^{n}$ where $\alpha$, the generator of $L \simeq \mathbb{Z}_{\ell}$, acts as a rotation by $-2 \pi / \ell$.

The following lemma is crucial for the numerical computation of equivariant Hopf points.

Lemma 4.7 Let the assumptions of the equivariant Hopf theorem 4.5 hold. If then $v+\mathrm{i} w \in X_{\ell}^{c}$ is an eigenvector of $\mathrm{D}_{x} f\left(x^{*}, \lambda^{*}\right)$ to the eigenvalue $\mathrm{i} \omega^{*}$ then $v+\mathrm{i} w=c\left(v^{*}+\mathrm{i} w^{*}\right)$ for some $c \in \mathbb{C}$ where $v^{*}+\mathrm{i} w^{*}$ is a Hopf eigenvector with $v^{*}, w^{*} \in N_{\omega^{*}}^{L}$.

Proof. The vector $v+\mathrm{i} w$ satisfies (4.9) and by the assumption of the equivariant Hopf theorem 4.5 there is only one such eigenvector (over $\mathbb{C}$ ), namely $v^{*}+\mathrm{i} w^{*}$. This proves the lemma.

Due to this lemma, we can solve (4.9) as follows: We first compute the space $X_{c}^{\ell}$. Then we compute the $L$-invariant Hopf point together with a Hopf eigenvector which lies in $X_{\ell}$ by an extended system.

First step of the algorithm. We compute an orthonormal basis of $X_{\ell}^{c}$ and store it as row vectors of a matrix $E_{\ell}$. We assume that $\ell>1$, i.e., that the branch of periodic orbits along which a Hopf bifurcation has been detected has non-trivial spatio-temporal symmetry $L=\mathbb{Z}_{\ell}$. We consider two cases:

Case 1: $\ell=2$. We compute the kernel $X_{2}=\operatorname{ker}\left(B_{2}\right)$ of $B_{2}=\alpha+\mathrm{id}_{n}$ and store an orthonormal basis of it in the row vectors of the $(d, n)$-matrix $E_{2}$. Here $d=\operatorname{dim} X_{2}$ and $X_{2}$ is the $L$-invariant subspace of $\mathbb{R}^{n}$ where the action of the symmetry group $L=\mathbb{Z}_{2}$ generated by $\alpha$ is given by $\left.\alpha\right|_{X_{2}}=-1$. Hence,

$$
E_{2}^{T} E_{2}=\left.\mathrm{id}\right|_{X_{2}}
$$

Case 2: $\ell>2$. In this case we compute the null space of the $(2 n, 2 n)$-matrix

$$
B_{\ell}:=\left(\begin{array}{cc}
\alpha-\cos (2 \pi / \ell) \mathrm{id}_{n} & -\sin (2 \pi / \ell) \mathrm{id}_{n} \\
\sin (2 \pi / \ell) \mathrm{id}_{n} & \alpha-\cos (2 \pi / \ell) \mathrm{id}_{n}
\end{array}\right) .
$$

We store an orthonormal basis of $\operatorname{ker}\left(B_{\ell}\right)$ in the row vectors of the matrix $E_{\ell}=\left[E_{V}, E_{W}\right] \in$ $\operatorname{Mat}(d, 2 n)$ where $E_{V}, E_{W} \in \operatorname{Mat}(d, n)$.

Before we continue with the description of the algorithm we present the following lemma: 
Lemma 4.8 Let $\ell>2$. Then the following holds true:

a) If $(v, w) \in \operatorname{ker} B_{\ell}, v, w \in \mathbb{R}^{n}$, we have $v+\mathrm{i} w \in X_{\ell}^{c}$.

b) If $(v, w) \in \operatorname{ker} B_{\ell}$, then so is $(-w, v)$. In particular, ker $B_{\ell}$ has even dimension $d=2 \hat{d}$, $\hat{d} \in \mathbb{N}$, the eigenvalue $\mathrm{e}^{-2 \pi \mathrm{i} / \ell}$ of $\alpha$ has geometric multiplicity $\hat{d}$, and $d=\operatorname{dim} X_{\ell}$.

c) For any $x \in \mathbb{R}^{d}$ we have $E_{V}^{T} x+\mathrm{i} E_{W}^{T} x \in X_{\ell}^{c}$.

d) Both $E_{V}^{T}$ and $E_{W}^{T}$ have $X_{\ell}$ as range.

\section{Proof.}

a) By Definition, $v+\mathrm{i} w \in X_{\ell}^{c}$ if and only if $v+\mathrm{i} w$ satisfies (4.10). Taking real and imaginary parts of the left and right hand side of (4.10) we see that $v+\mathrm{i} w$ satisfies $(4.10)$ iff $(v, w) \in$ ker $B_{\ell}$.

b) follows from a) and the fact that $(v, w) \simeq v+\mathrm{i} w$ and $\mathrm{i}(v+\mathrm{i} w) \sim(-w, v)$ are linearly independent over $\mathbb{R}$.

c) follows from the definition of $E_{\ell}$.

d) follows from a) and b).

Second step of the algorithm. Again we consider two cases: $\ell=2, \ell>2$.

Case 1: $\ell=2$. We solve an extended system

$$
F\left(x_{\text {red }}, \lambda, v_{\text {red }}, w_{\text {red }}, \omega\right)=0,
$$

similarly to (4.2), where now

$$
v_{\text {red }}, w_{\text {red }} \in X_{\ell} \simeq \mathbb{R}^{d}, \quad x_{\text {red }} \in \operatorname{Fix}(L) \simeq \mathbb{R}^{n_{\text {red }}}
$$

and

$$
F: \mathbb{R}^{n_{\text {red }}+2 d+2} \rightarrow \mathbb{R}^{n_{\text {red }}+2 d+1} .
$$

Let $f_{\text {red }}:=\left.f\right|_{\text {Fix }(L)}$ and let $Q: \mathbb{R}^{n} \rightarrow \mathbb{R}^{n_{\text {red }}}$ be the matrix which contains an orthonormal basis of $\operatorname{Fix}(L), \operatorname{dim} \operatorname{Fix}(L)=n_{\text {red }}$, as row vectors. Then we define $F$ as

$$
F\left(x_{\text {red }}, \lambda, v_{\text {red }}, w_{\text {red }}, \omega\right)=\left(\begin{array}{l}
f_{\text {red }}\left(x_{\text {red }}, \lambda\right) \\
\left.E_{2} \mathrm{D}_{x} f(x, \lambda)\right|_{x=Q^{T} x_{\text {red }} E_{2}^{T} v_{\text {red }}+\omega w_{\text {red }}} \\
-\omega v_{\text {red }}+\left.E_{2} \mathrm{D}_{x} f(x, \lambda)\right|_{x=Q^{T} x_{\text {red }}} E_{2}^{T} w_{\text {red }} \\
\left\langle v_{\text {red }}, v_{\text {red }}\right\rangle+\left\langle w_{\text {red }}, w_{\text {red }}\right\rangle-1
\end{array}\right) .
$$

Case 2: $\ell>2$. In this case the real part $v^{*}$ of the Hopf eigenvector $v^{*}+\mathrm{i} w^{*}$ and the knowledge of the drift symmetry $\alpha$ of the branch of periodic orbits along which a Hopf bifurcation was detected determines the Hopf eigenvector uniquely since $v^{*}+\mathrm{i} w^{*}$ satisfies (4.10) and so

$$
w^{*}=\frac{1}{\sin (2 \pi / \ell)}\left(\alpha v^{*}-\cos (2 \pi / \ell) v^{*}\right) .
$$


Therefore only the real part of the Hopf eigenvector, the Hopf point, its parameter and the Hopf frequency need to be computed by an extended system. We define $F: \mathbb{R}^{n_{\text {red }}+d+2} \rightarrow \mathbb{R}^{n_{\text {red }}+d+1}$ by

$$
F\left(x_{\text {red }}, \lambda, v_{\text {red }}, \omega\right)=\left(\begin{array}{l}
f_{\text {red }}\left(x_{\text {red }}, \lambda\right) \\
E_{V}\left(\left.\mathrm{D}_{x} f(x, \lambda)\right|_{x=Q^{T} x_{\text {red }}} E_{V}^{T} v_{\text {red }}+\omega E_{W}^{T} v_{\text {red }}\right) \\
\left\langle v_{\text {red }}, v_{\text {red }}\right\rangle-1
\end{array}\right) .
$$

\section{Proposition 4.9}

a) If $\ell=2$ and $\left(x_{\mathrm{red}}^{*}, \lambda^{*}, v_{\mathrm{red}}^{*}, w_{\mathrm{red}}^{*}, \omega^{*}\right)$ is a solution to $F=0$ as defined in (4.13) then $x^{*}=Q^{T} x_{\mathrm{red}}^{*}$ is an equivariant Hopf point with Hopf frequency $\omega^{*}$. Moreover a Hopf eigenvector $v^{*}+\mathrm{i} w^{*}$ with $v^{*}, w^{*} \in N_{\omega^{*}}^{L}$ is given by $v^{*}=E_{2}^{T} v_{\mathrm{red}}^{*}, w^{*}=E_{2}^{T} w_{\mathrm{red}}^{*}$.

b) Similarly, if $\ell>2$, and $\left(x_{\mathrm{red}}^{*}, \lambda^{*}, v_{\mathrm{red}}^{*}, \omega^{*}\right)$ is a solution to $F=0$ as defined in (4.14) then $x^{*}=Q^{T} x_{\mathrm{red}}^{*}$ is an equivariant Hopf point with Hopf frequency $\omega^{*}$. Moreover a Hopf eigenvector $v^{*}+\mathrm{i} w^{*}$ with $v^{*}, w^{*} \in N_{\omega^{*}}^{L}$ is given by $v^{*}=E_{V}^{T} v_{\mathrm{red}}^{*}, w^{*}=E_{W}^{T} v_{\mathrm{red}}^{*}$.

For the proof we need the following lemma:

Lemma 4.10 Let $A$ be an $(n, n)$-matrix which is equivariant with respect to a linear $\mathbb{Z}_{\ell \text {-action }}$ on $\mathbb{R}^{n}$. Let $\alpha$ generate $\mathbb{Z}_{\ell}$ and define $X_{\ell}$ as before. Then $X_{\ell}$ is A-invariant.

Proof. Let $v \in X_{\ell}$. Then there is some $w \in X_{\ell}$ such that $v+\mathrm{i} w$ is an eigenvector of $\alpha$ to the eigenvalue $\exp (-2 \pi \mathrm{i} / \ell)$. By $\mathbb{Z}_{\ell}$-equivariance of $A$ also $A(v+\mathrm{i} w)$ is an eigenvector of $\alpha$ to the eigenvalue $\exp (-2 \pi \mathrm{i} / \ell)$ and so both $A v$ and $A w$ lie in $X_{\ell}$.

Note that $X_{\ell}$ is an isotypic component of the $\mathbb{Z}_{\ell}$ action on $\mathbb{R}^{n}$, and that generally isotypic components for a linear action of a group $\Gamma$ are invariant under $\Gamma$-equivariant matrices [11].

\section{Proof of Proposition 4.9.}

a) Case $\ell=2$. The first equation $f_{\text {red }}(x, \lambda)=0$ of $F=0$ implies that $x_{\mathrm{red}}^{*}$ is an equilibrium of $f_{\text {red }}\left(\cdot, \lambda^{*}\right)$. Hence $x^{*}=Q^{T} x_{\text {red }}^{*}$ is an $L$-invariant equilibrium of $f\left(\cdot, \lambda^{*}\right)$. From the other equations in $F=0$ we conclude that $v_{\text {red }}^{*}+\mathrm{i} w_{\text {red }}^{*}$ is an eigenvector of $E_{2} \mathrm{D}_{x} f\left(x^{*}, \lambda^{*}\right) E_{2}^{T}$ to the eigenvalue $\mathrm{i} \omega^{*}$. Since $x^{*} \in \operatorname{Fix}(L)$ the derivative $\mathrm{D}_{x} f\left(x^{*}, \lambda^{*}\right)$ is $L$-equivariant by Lemma 4.3 and therefore, by Lemma 4.10, maps $X_{2}$ into itself. Let $v^{*}=E_{2}^{T} v_{\text {red }}^{*}$, $w^{*}=E_{2}^{T} w_{\mathrm{red}}^{*}$. Because of (4.11), $v^{*}+\mathrm{i} w^{*} \in X_{2}^{c}$ is an eigenvector of $\mathrm{D}_{x} f\left(x^{*}, \lambda^{*}\right)$ to the eigenvalue $\mathrm{i} \omega^{*}$. Hence $\left(x^{*}=Q^{T} x_{\text {red }}^{*}, \lambda^{*}\right)$ is a Hopf point. Lemma 4.7 now implies that $v^{*}, w^{*} \in N_{\omega^{*}}^{L}$.

b) Case $\ell>2$. As in the case $\ell=2$ the first equation of $F=0$ implies that $x^{*}=Q^{T} x_{\text {red }}^{*}$ is an $L$-invariant equilibrium of $f\left(\cdot, \lambda^{*}\right)$. Let $v^{*}=E_{V}^{T} v_{\mathrm{red}}^{*}$ and $w^{*}=E_{W}^{T} w_{\mathrm{red}}^{*}$. From Lemma 4.8 c) we conclude that $v^{*}+\mathrm{i} w^{*} \in X_{\ell}^{c}$. Since $\mathrm{D}_{x} f\left(Q^{T} x_{\text {red }}(\lambda), \lambda\right)$ is $L$-equivariant by Lemma 4.3 and hence maps $X_{\ell}$ into itself by Lemma 4.10 and since $\left.E_{V}\right|_{X_{\ell}}$ is an isomorphism by Lemma 4.8 d), the other equations in $F=0$ imply that

$$
\mathrm{D}_{x} f\left(x^{*}, \lambda^{*}\right) v^{*}+\omega^{*} w^{*}=0
$$

and so

$$
\operatorname{Re}\left(\mathrm{D}_{x} f\left(x^{*}, \lambda^{*}\right)\left(v^{*}+\mathrm{i} w^{*}\right)-\mathrm{i} \omega^{*}\left(v^{*}+\mathrm{i} w^{*}\right)\right)=0 .
$$

Multiplying (4.15) by $\alpha$ and using the $L$-equivariance of $\mathrm{D}_{x} f\left(x^{*}, \lambda^{*}\right)$ we obtain

$$
\mathrm{D}_{x} f\left(x^{*}, \lambda^{*}\right) \alpha v^{*}+\omega^{*} \alpha w^{*}=0 .
$$


From the fact that $v^{*}+\mathrm{i} w^{*} \in X_{\ell}^{c}$ we conclude

$$
\operatorname{Re}\left(\mathrm{e}^{-2 \pi \mathrm{i} / \ell}\left(\mathrm{D}_{x} f\left(x^{*}, \lambda^{*}\right)\left(v^{*}+\mathrm{i} w^{*}\right)-\mathrm{i} \omega^{*}\left(v^{*}+\mathrm{i} w^{*}\right)\right)\right)=0 .
$$

Since $\mathrm{e}^{2 \pi \mathrm{i} / \ell} \notin \mathbb{R}$ this implies that

$$
\mathrm{D}_{x} f\left(x^{*}, \lambda^{*}\right)\left(v^{*}+\mathrm{i} w^{*}\right)=\mathrm{i} \omega^{*}\left(v^{*}+\mathrm{i} w^{*}\right)
$$

and therefore $\left(x^{*}, \lambda^{*}\right)$ is a Hopf point and $v^{*}+\mathrm{i} w^{*} \in X_{\ell}^{c}$ a Hopf eigenvector. By Lemma 4.7 we then get $v^{*}, w^{*} \in N_{\omega^{*}}^{L}$.

Analogously to Theorem 4.2 we have:

Theorem 4.11 If the assumptions of the equivariant Hopf Theorem 4.5 hold and if the initial guess is good enough then the Gauss-Newton method applied to (4.13) for $\ell=2$ and applied to (4.14) for $\ell>2$ converges.

Proof. Similarly as in the non-symmetric case (Theorem 4.2, see $[1,12,14]$ ) we show that DF, with $F$ from (4.13) for $\ell=2$ and from (4.14) for $\ell>2$, has full rank in the Hopf point. As before we consider the cases $\ell=2$ and $\ell>2$ separately.

Case $\ell=2$. The assumptions of the equivariant Hopf theorem imply that $\mathrm{i} \omega^{*}$ is a simple eigenvalue of $\left.\mathrm{D}_{x} f\left(x^{*}, \lambda^{*}\right)\right|_{X_{2}}$ and hence, due to (4.11), also of $E_{2} \mathrm{D}_{x} f\left(x^{*}, \lambda^{*}\right) E_{2}^{T}$. The proof that $\mathrm{D} F$ has full rank in the Hopf point is therefore very similar to the non-symmetric case: the only difference is that we require $v, w \in X_{2}$ and $x \in \operatorname{Fix}(L)$. We omit the details.

Case $\ell>2$. In this case we have

$$
\begin{aligned}
& \mathrm{D}_{\left(x_{\text {red }}, \lambda, v_{\text {red }}, \omega\right)} F\left(x_{\text {red }}, \lambda, v_{\text {red }}, \omega\right)= \\
& \left(\begin{array}{cccc}
\mathrm{D}_{x_{\text {ree }}} f_{\text {red }}\left(x_{\text {red }}, \lambda\right) & \mathrm{D}_{\lambda} f_{\text {red }}\left(x_{\text {red }}, \lambda\right) & 0 & 0 \\
E_{V} \mathrm{D}_{x}^{2} f E_{V}^{T} v_{\text {red }} Q^{T} & E_{V} \mathrm{D}_{x} \mathrm{D}_{\lambda} f E_{V}^{T} v_{\text {red }} & E_{V}\left(\mathrm{D}_{x} f E_{V}^{T}+\omega E_{W}^{T}\right) & E_{V} E_{W}^{T} v_{\text {red }} \\
0 & 0 & 2 v_{\text {red }}^{T} & 0
\end{array}\right),
\end{aligned}
$$

where $f$ is short for $f\left(Q^{T} x_{\text {red }}, \lambda\right)$. Let $t=\left(t_{x_{\text {red }}}, t_{\lambda}, t_{v_{\text {red }}}, t_{\omega}\right)$ be a kernel vector of $\mathrm{D} F$ in the equivariant Hopf point:

$$
\begin{aligned}
0= & \mathrm{D}_{x_{\mathrm{red}}} f_{\mathrm{red}}\left(x_{\mathrm{red}}^{*}, \lambda^{*}\right) t_{x_{\mathrm{red}}}+\mathrm{D}_{\lambda} f_{\mathrm{red}}\left(x_{\mathrm{red}}^{*}, \lambda^{*}\right) t_{\lambda}, \\
0 & E_{V} \mathrm{D}_{x}^{2} f E_{V}^{T} v_{\mathrm{red}}^{*} Q^{T} t_{x_{\mathrm{red}}}+E_{V} \mathrm{D}_{x} \mathrm{D}_{\lambda} f E_{V}^{T} v_{\mathrm{red}}^{*} t_{\lambda} \\
& +E_{V}\left(\mathrm{D}_{x} f E_{V}^{T}+\omega^{*} E_{W}^{T}\right) t_{v_{\mathrm{red}}}+E_{V} E_{W}^{T} v_{\mathrm{red}}^{*} t_{\omega}, \\
0= & 2\left\langle v_{\mathrm{red}}^{*}, t_{v_{\mathrm{red}}}\right\rangle .
\end{aligned}
$$

We need to show that $\operatorname{ker}(\mathrm{D} F)$ is one-dimensional. Similarly, as in the proof of convergence for the non-symmetric case, Theorem 4.2, see e.g. $[1,12,14]$, we conclude from the first equation of (4.16) that

$$
\left(t_{x_{\mathrm{red}}}, t_{\lambda}\right)=t_{\lambda}\left(x_{\mathrm{red}}^{\prime}\left(\lambda^{*}\right), 1\right) .
$$

Since

$$
\mathrm{D}_{x}^{2} f\left(x^{*}, \lambda^{*}\right) x^{\prime}\left(\lambda^{*}\right)+\mathrm{D}_{x} \mathrm{D}_{\lambda} f\left(x^{*}, \lambda^{*}\right)=\left.\frac{\mathrm{d}}{\mathrm{d} \lambda} \mathrm{D}_{x} f(x(\lambda), \lambda)\right|_{\lambda=\lambda^{*}},
$$


where $x(\lambda)=Q^{T} x_{\text {red }}(\lambda)$, we deduce from the second equation of (4.16) that

$$
E_{V}\left(\left.t_{\lambda} \frac{\mathrm{d}}{\mathrm{d} \lambda} \mathrm{D}_{x} f(x(\lambda), \lambda)\right|_{\lambda=\lambda^{*}} E_{V}^{T} v_{\mathrm{red}}^{*}+\left(\mathrm{D}_{x} f\left(x^{*}, \lambda^{*}\right) E_{V}^{T}+\omega^{*} E_{W}^{T}\right) t_{v_{\mathrm{red}}}+t_{\omega} E_{W}^{T} v_{\mathrm{red}}^{*}\right)=0 .
$$

Since $E_{V}^{T} v_{\mathrm{red}}^{*}, E_{V}^{T} t_{v}, E_{W}^{T} v_{\mathrm{red}}^{*}, E_{W}^{T} t_{v} \in X_{\ell}$ and $\left.E_{V}\right|_{X_{\ell}}$ is an isomorphism by Lemma $\left.4.8 \mathrm{~d}\right)$ and since $\mathrm{D}_{x} f(x(\lambda), \lambda)$ maps $X_{\ell}$ into itself by Lemmata 4.3 and $4.10,(4.17)$ implies that

$$
\left.t_{\lambda} \frac{\mathrm{d}}{\mathrm{d} \lambda} \mathrm{D}_{x} f(x(\lambda), \lambda)\right|_{\lambda=\lambda^{*}} E_{V}^{T} v_{\mathrm{red}}^{*}+\mathrm{D}_{x} f\left(x^{*}, \lambda^{*}\right) E_{V}^{T} t_{v}+\omega^{*} E_{W}^{T} t_{v}+t_{\omega} E_{W}^{T} v_{\mathrm{red}}^{*}=0 .
$$

Let $t_{v}=E_{V}^{T} t_{v_{\text {red }}}$ and $t_{w}=E_{W}^{T} t_{v_{\text {red }}}$. By Lemma $4.8 \mathrm{c}$ ) we have $t_{v}+\mathrm{i} t_{w} \in X_{\ell}^{c}$ which we will need later on. Denote $v^{*}=E_{V}^{T} v_{\text {red }}^{*}, w^{*}=E_{W}^{T} v_{\text {red }}^{*}$ such that, by Proposition $\left.4.9 \mathrm{~b}\right), v^{*}+\mathrm{i} w^{*}$ is a Hopf eigenvector. Then we get

$$
\begin{aligned}
0= & \left.t_{\lambda} \frac{\mathrm{d}}{\mathrm{d} \lambda} \mathrm{D}_{x} f(x(\lambda), \lambda)\right|_{\lambda=\lambda^{*}} v^{*}+\mathrm{D}_{x} f\left(x^{*}, \lambda^{*}\right) t_{v}+\omega^{*} t_{w}+t_{\omega} w^{*} \\
= & \operatorname{Re}\left(\left.t_{\lambda} \frac{\mathrm{d}}{\mathrm{d} \lambda} \mathrm{D}_{x} f(x(\lambda), \lambda)\right|_{\lambda=\lambda^{*}}\left(v^{*}+\mathrm{i} w^{*}\right)+\mathrm{D}_{x} f\left(x^{*}, \lambda^{*}\right)\left(t_{v}+\mathrm{i} t_{w}\right)\right) \\
& -\operatorname{Re}\left(\mathrm{i} \omega^{*}\left(t_{v}+\mathrm{i} t_{w}\right)+\mathrm{i} t_{\omega}\left(v^{*}+\mathrm{i} w^{*}\right)\right) .
\end{aligned}
$$

By Lemma $4.8 \mathrm{c}), v^{*}+\mathrm{i} w^{*} \in X_{\ell}^{c}$. Therefore

$$
\begin{aligned}
0= & \left.\alpha t_{\lambda} \frac{\mathrm{d}}{\mathrm{d} \lambda} \mathrm{D}_{x} f(x(\lambda), \lambda)\right|_{\lambda=\lambda^{*}} v^{*}+\alpha \mathrm{D}_{x} f\left(x^{*}, \lambda^{*}\right) t_{v}+\omega^{*} \alpha t_{w}+t_{\omega} \alpha w^{*} \\
= & \left.t_{\lambda} \frac{\mathrm{d}}{\mathrm{d} \lambda} \mathrm{D}_{x} f(x(\lambda), \lambda)\right|_{\lambda=\lambda^{*}} \alpha v^{*}+\mathrm{D}_{x} f\left(x^{*}, \lambda^{*}\right) \alpha t_{v}+\omega^{*} \alpha t_{w}+t_{\omega} \alpha w^{*} \\
= & \operatorname{Re}\left(\mathrm{e}^{-2 \pi \mathrm{i} / \ell}\left(\left.t_{\lambda} \frac{\mathrm{d}}{\mathrm{d} \lambda} \mathrm{D}_{x} f(x(\lambda), \lambda)\right|_{\lambda=\lambda^{*}}\left(v^{*}+\mathrm{i} w^{*}\right)+\mathrm{D}_{x} f\left(x^{*}, \lambda^{*}\right)\left(t_{v}+\mathrm{i} t_{w}\right)\right)\right) \\
& -\operatorname{Re}\left(\mathrm{e}^{-2 \pi \mathrm{i} / \ell}\left(\mathrm{i} \omega^{*}\left(t_{v}+\mathrm{i} t_{w}\right)-\mathrm{i} t_{\omega}\left(v^{*}+\mathrm{i} w^{*}\right)\right)\right)
\end{aligned}
$$

where we used the $L$-equivariance of $\mathrm{D}_{x} f(x(\lambda), \lambda)$ (Lemma 4.3) in the second line. Since $\mathrm{e}^{-2 \pi \mathrm{i} / \ell} \notin$ $\mathbb{R}$ for $\ell>2$ these last two equations imply that

$$
\begin{aligned}
0= & \left.t_{\lambda} \frac{\mathrm{d}}{\mathrm{d} \lambda} \mathrm{D}_{x} f(x(\lambda), \lambda)\right|_{\lambda=\lambda^{*}}\left(v^{*}+\mathrm{i} w^{*}\right) \\
& +\mathrm{D}_{x} f\left(x^{*}, \lambda^{*}\right)\left(t_{v}+\mathrm{i} t_{w}\right)-\mathrm{i} \omega^{*}\left(t_{v}+\mathrm{i} t_{w}\right)-\mathrm{i} t_{\omega}\left(v^{*}+\mathrm{i} w^{*}\right) .
\end{aligned}
$$

Let $v(\lambda)+\mathrm{i} w(\lambda), v\left(\lambda^{*}\right)=v^{*}, w\left(\lambda^{*}\right)=w^{*}, v(\lambda), w(\lambda) \in X_{\ell}$, be the path of eigenvectors of $\mathrm{D}_{x} f(x(\lambda), \lambda)$ to the eigenvalues $\mu(\lambda)$ with $\|v(\lambda)\|=1$. Let $u^{*}=v^{*}+\mathrm{i} w^{*}$ and let $u_{L}^{*} \in X_{\ell}^{c}$ be the uniquely determined left eigenvector of $\mathrm{D}_{x} f\left(x^{*}, \lambda^{*}\right)$ to the eigenvalue $i \omega^{*}$ with $\left\langle u_{L}^{*}, u^{*}\right\rangle=1$. As in the non-symmetric case, see $[1,14,12],(4.18)$ then implies that

$$
t_{\lambda} u_{L}^{*} \frac{\mathrm{d}}{\mathrm{d} \lambda} \mathrm{D}_{x} f(x(\lambda), \lambda)_{\lambda=\lambda^{*}} u^{*}=\mathrm{i} t_{\omega} .
$$

This combined with

$$
\left\langle u_{L}^{*}, \frac{\mathrm{d}}{\mathrm{d} \lambda} \mathrm{D}_{x} f(x(\lambda), \lambda)_{\lambda=\lambda^{*}} u^{*}\right\rangle=\mu^{\prime}\left(\lambda^{*}\right)
$$

and the assumption that $\operatorname{Re} \mu^{\prime}\left(\lambda^{*}\right) \neq 0$ gives $t_{\lambda}=0$. Hence $t_{x}=0$ and $t_{\omega}=0$. As in the proof of Theorem 4.2, (4.18) reduces to

$$
\mathrm{D}_{x} f\left(x^{*}, \lambda^{*}\right)\left(t_{v}+\mathrm{i} t_{w}\right)-\mathrm{i} \omega^{*}\left(t_{v}+\mathrm{i} t_{w}\right)=0
$$


so that $t_{v}+\mathrm{i} t_{w}$ is a Hopf eigenvector. Since $t_{v}+\mathrm{i} t_{w} \in X_{\ell}^{c}$ we deduce from Lemma 4.7 that $t_{v}+\mathrm{i} t_{w}=c\left(v^{*}+\mathrm{i} w^{*}\right)$ for some $c \in \mathbb{C}$. By the definition of $v^{*}, w^{*}$ and $t_{v}, t_{w}$ this is equivalent to

$$
E_{V}^{T} t_{v_{\text {red }}}+\mathrm{i} E_{W}^{T} t_{v_{\text {red }}}=c\left(E_{V}^{T} v_{\text {red }}^{*}+\mathrm{i} E_{W}^{T} v_{\text {red }}^{*}\right) .
$$

By Lemma 4.8 the map $E_{V} E_{V}^{T} \in \operatorname{Mat}(d)$ is invertible. Therefore we conclude from (4.19) that $t_{v_{\text {red }}} \in \operatorname{span}\left(v_{\text {red }}^{*}, w_{\text {red }}^{*}\right)$, where $w_{\text {red }}^{*}:=\left(E_{V} E_{V}^{T}\right)^{-1} E_{V} E_{W}^{T} v_{\text {red }}^{*}$. From the last row of $\mathrm{D} F$ we get the additional condition $\left\langle v_{\text {red }}^{*}, t_{v_{\text {red }}}\right\rangle=0$ on $t_{v_{\text {red }}}$. Hence the kernel of $\mathrm{D} F$ is one-dimensional and $\mathrm{D} F$ has full rank in the Hopf point.

Initial guess for the Gauss-Newton iteration As in the non-symmetric case, see Section 4.1.2, we use Hermite interpolation between two consecutive periodic orbits $y^{(0)}$ and $y^{(1)}$ between of which a Hopf bifurcation was detected and determine the point $\hat{\tau}$ on the interpolating polynomial $y(\tau)=(x(\tau), T(\tau), \lambda(\tau))$ with $\lambda^{\prime}(\hat{\tau})=0$. This way we obtain an initial approximation $\hat{x}=x(\hat{\tau})$ for the Hopf point, an initial approximation $\hat{\lambda}=\lambda(\hat{\tau})$ for its parameter, and an initial approximation $\hat{\omega}=\frac{2 \pi}{\hat{T}}$ for its frequency, where $\hat{T}=T(\hat{\tau})$. If $\ell=2$ we let

$$
\hat{v}=c E_{2} \frac{\mathrm{d}}{\mathrm{d} \tau} x_{1}(\hat{\tau}), \quad \hat{w}=-c \frac{1}{\hat{\omega}} \mathrm{D}_{x} f(\hat{x}, \hat{\lambda}) \frac{\mathrm{d}}{\mathrm{d} \tau} x_{1}(\hat{\tau})
$$

where $c$ is such that $\langle\hat{v}, \hat{v}\rangle+\langle\hat{w}, \hat{w}\rangle=1$ and take the point $\left(\hat{x}_{\text {red }}=Q \hat{x}, \hat{\lambda}, \hat{v}, \hat{w}, \hat{\omega}\right)$ as initial guess for the Gauss-Newton iteration applied to (4.13). If $\ell>2$ we define

$$
\hat{v}=c E_{V} \frac{\mathrm{d}}{\mathrm{d} \tau} x_{1}(\hat{\tau})
$$

with $c \in \mathbb{R}$ such that $\langle\hat{v}, \hat{v}\rangle=1$ and use $\left(\hat{x}_{\text {red }}, \hat{\lambda}, \hat{v}, \hat{\omega}\right)$ as initial guess for the Gauss-Newton iteration applied to (4.14).

\section{Applications}

In this section we illustrate the numerical methods for the continuation of symmetric periodic orbits which we presented in the preceding sections and implemented in the code SYMPERCON [20] with some examples.

\subsection{The Lorenz model - comparison with AUTO and CONTENT}

There are many programs for the numerical continuation of periodic orbits of non-symmetric systems. Two of the most well known and widely used programs are AUTO [8] and CONTENT [17] the numerical part of which is based on AUTO. In both programs collocation is used to find periodic solutions. This approach is equivalent to the multiple shooting ansatz with the multiple shooting points being the grid points and the IVP solver having only one step.

One of the key new features of SYMPERCON compared to those program packages is the exploitation of symmetries of periodic orbits and the computation of symmetry breaking and symmetry increasing bifurcations, and we present some applications of these methods in the next sections. In this section we give numerical evidence that the program package SYMPERCON is also competitive when applied to continuation of non-symmetric periodic orbits. We compare the programs AUTO, CONTENT and SYMPERCON using an example which is taken from 

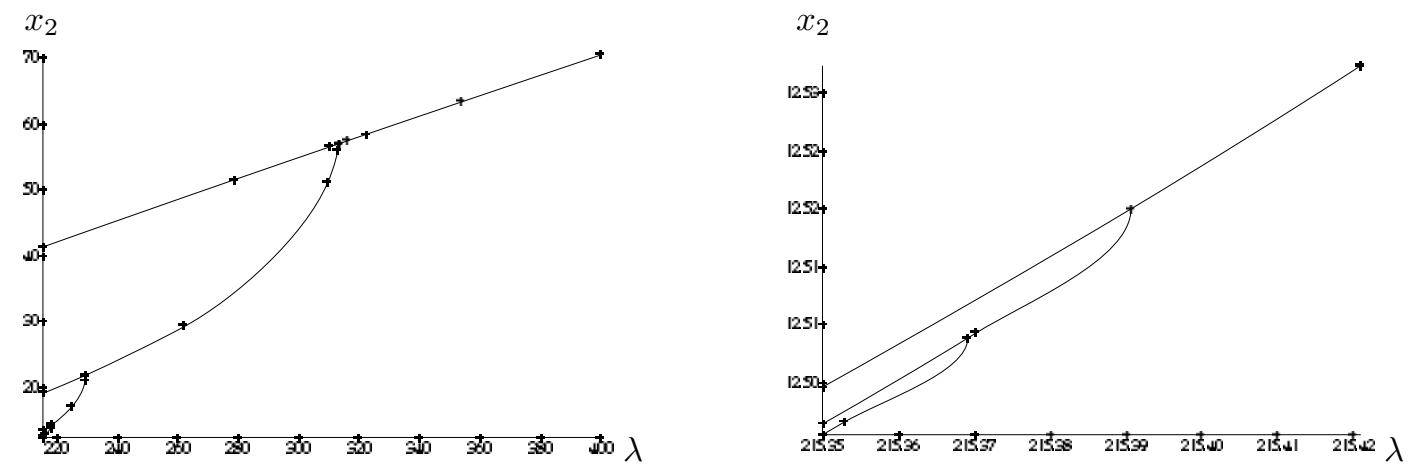

Figure 4: Feigenbaum cascade in the Lorenz model as computed by SYMPERCON. The right figure is a zoom of the lower left corner of the left figure.

the CONTENT Tutorial (ver 1.4) [17] "ODEs: Lorenz system: Continuation of limit cycles and branch switching". Starting with the Lorenz system

$$
\begin{aligned}
x_{1}^{\prime} & =-\sigma x_{1}+\sigma x_{2}, \\
x_{2}^{\prime} & =-x_{1} x_{3}+\lambda x_{1}-x_{2} . \\
x_{3}^{\prime} & =x_{1} x_{2}-b x_{3},
\end{aligned}
$$

where

$$
\sigma=10, b=8 / 3
$$

all programs find a periodic solution for the initial guess

$$
\left(x^{*}, T^{*}, \lambda^{*}\right)=((16.2,57.4,250.8), 0.411,312)
$$

from [17]. We continue this limit cycle with respect to the parameter $\lambda$ with required relative error tol $=10^{-5}$. The Lorenz system has the $\mathbb{Z}_{2}$-symmetry $\left(x_{1}, x_{2}, x_{3}\right) \rightarrow\left(-x_{1},-x_{2}, x_{3}\right)$ and the above periodic orbit is $\mathbb{Z}_{2}$-symmetric. All programs detect a bifurcation of this periodic orbit at $\lambda=312.97$. This is a symmetry breaking flip pitchfork bifurcation, so the bifurcating periodic orbits are non-symmetric. The bifurcating branch of non-symmetric periodic orbits which we take as primary branch in this comparison subsequently undergoes a period doubling cascade, and we compared AUTO, CONTENT and SYMPERCON in their performance computing this bifurcation cascade.

While SYMPERCON automatically doubles the number of multiple shooting points at each flip bifurcation up to a given maximum, in AUTO and CONTENT the entered number of grid points has to be set by the user. Since this number of grid points has to be increased at period doubling bifurcations - otherwise the Newton method fails to converge - we doubled the number manually. But after the 4 th period doubling we did not manage to configure CONTENT in such a way that it would find any more period doubling points. With AUTO we found a 5th period doubling bifurcation. In comparison, SYMPERCON found periodic orbits of $2^{6}$ times the original period, with period-doubling bifurcations starting from the primary branch at parameters $\lambda=229.41, \lambda=218.21, \lambda=215.97, \lambda=215.49, \lambda=215.39$, and $\lambda=215.37$, see Figure 4, and (when run with the compiler gcc under linux) even a 7 th period doubling point at $\lambda=215.36$. But all programs were very sensitive to changes of the parameters of the computation (like the number of initial grid points $m$, the initial continuation steplength etc) and the choice of $\mathrm{C}$ compiler used. 


\subsection{Symmetry breaking bifurcations in coupled cells}

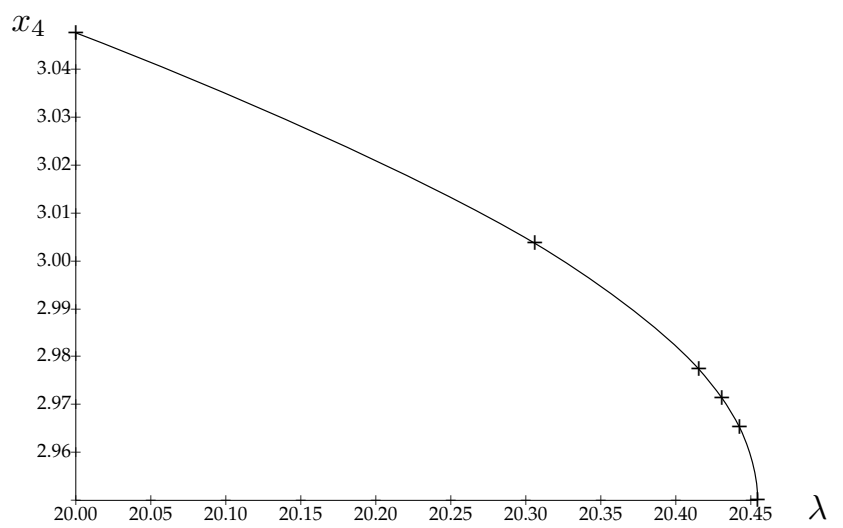

Figure 5: Equivariant Hopf bifurcation of $\mathbb{Z}_{4}$-symmetric periodic orbits of the Brusselator

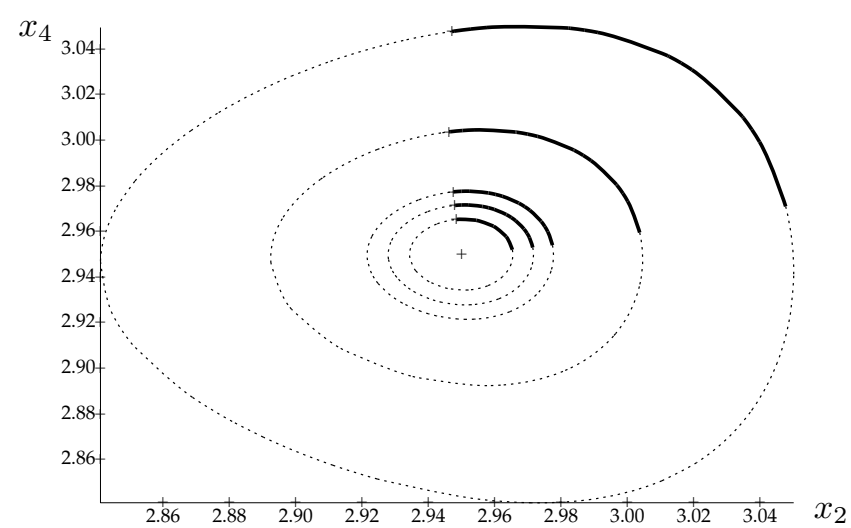

Figure 6: $\mathbb{Z}_{4}$-symmetric periodic orbits near an equivariant Hopf point of the Brusselator - the bold parts of the trajectories are computed numerically.

In this section we apply our methods to compute equivariant Hopf points and symmetry breaking flip pitchfork bifurcations in the Brusselator model of coupled cells with parameters as in [3]. We consider four identical cells in which the same reaction takes place and which are coupled by diffusion. The equations for the 4 -cell Brusselator are the following $(j=1,3,5,7$, $x_{i}:=x_{i-8}$ for $\left.i>8\right)$

$$
\begin{aligned}
x_{j}^{\prime} & =A-(B+1) x_{j}+x_{j}^{2} x_{j+1}+\lambda\left(-3 x_{j}+x_{j+2}+x_{j+4}+x_{j+6}\right) / 1000, \\
x_{j+1}^{\prime} & =B x_{j}-x_{j}^{2} x_{j+1}+\lambda\left(-3 x_{j+1}+x_{j+3}+x_{j+5}+x_{j+7}\right) / 1000,
\end{aligned}
$$

where $A=2.0, B=5.9$. The problem is invariant with respect to permutations of the cells, thus the equations are $S_{4}$-equivariant. We denote the elements of $\Gamma=S_{4}$ by $\gamma_{i j k l}$, e.g.,

$$
\gamma_{2314}=\left(\begin{array}{cccc}
0 & 0 & \text { id } & 0 \\
\text { id } & 0 & 0 & 0 \\
0 & \text { id } & 0 & 0 \\
0 & 0 & 0 & \text { id . }
\end{array}\right), \quad \text { id } \in \operatorname{Mat}(2,2) .
$$


From [3] we took the following equivariant Hopf point

$$
\left(x^{*}, \lambda^{*}\right)=(2.0,2.95,2.0,2.95,2.0,2.95,2.0,2.95,20.45) .
$$

The corresponding imaginary eigenvalue $\omega^{*} \mathrm{i}$ has the value $\omega^{*}=0.62058$ and belongs to an absolutely irreducible 3 -dimensional representation, c.f. Remark 2.4. The real eigenspace $N_{\omega^{*}}$ of $\omega^{*} \mathrm{i}$ is of the form $N_{\omega^{*}}=V \oplus W$ with

$$
\begin{aligned}
V & =\left\{\left(v_{1}, 0, v_{2}, 0, v_{3}, 0,-v_{1}-v_{2}-v_{3}, 0\right) \mid v_{1}, v_{2}, v_{3} \in \mathbb{R}\right\} \\
W & =\left\{\left(0, w_{1}, 0, w_{2}, 0, w_{3}, 0,-w_{1}-w_{2}-w_{3}\right) \mid w_{1}, w_{2}, w_{3} \in \mathbb{R}\right\} .
\end{aligned}
$$

Let $e_{j}^{V}, e_{j}^{W}$ be the $j$-th unit vector of $V$ and $W$ respectively. Then the vectors $e_{j}^{V}+\mathrm{i} e_{j}^{W}$, $j=1,2,3$, are eigenvectors to the Hopf eigenvalues $i \omega^{*}$ :

$$
\mathrm{D}_{x} f\left(x^{*}, \lambda^{*}\right)\left(e_{j}^{V}+\mathrm{i} e_{j}^{W}\right)=\mathrm{i} \omega^{*}\left(e_{j}^{V}+\mathrm{i} e_{j}^{W}\right), \quad j=1, \ldots, 3 .
$$

Using the equivariant Hopf Theorem (Theorem 4.5) we can now compute the symmetries and initial planes of the emanating periodic solutions, as in [3]. As an example we consider periodic orbits with spatio-temporal symmetry group

$$
L=\left\{\mathrm{id},\left(\gamma_{3142}, \frac{3}{4}\right),\left(\gamma_{4321}, \frac{1}{2}\right),\left(\gamma_{2413}, \frac{1}{4}\right)\right\}
$$

which means

$$
L=\mathbb{Z}_{4}, K=\{\mathrm{id}\} .
$$

In order to compute the starting plane $N_{\omega^{*}}^{L}$ we need to know how $S^{1}$ acts on $N_{\omega^{*}}$. Let $u=$ $(v, w) \in N_{\omega^{*}}=V \oplus W$. Due to (5.1) and (4.6) we have for $\theta \in S^{1} \simeq \mathbb{R} / \mathbb{Z}$

$$
(\mathrm{id}, \theta) u=\left(\begin{array}{c}
\cos (2 \pi \theta) v-\sin (2 \pi \theta) w \\
\sin (2 \pi \theta) v+\cos (2 \pi \theta) w
\end{array}\right)
$$

By the equivariant Hopf Theorem 4.5 the starting plane $N_{\omega^{*}}^{L}$ is determined by the condition

$$
N_{\omega^{*}}^{L}=\left\{u \in N_{\omega^{*}} \mid(\gamma, \theta) u=u \forall(\gamma, \theta) \in L\right\}=\left\{u \in N_{\omega^{*}} \mid\left(\gamma_{2413}, \frac{\pi}{2}\right) u=u\right\} .
$$

Here we used that $L=\mathbb{Z}_{4}$ is generated by $\gamma_{2413}$. After a short computation we obtain

$$
N_{\omega^{*}}^{L}=\left\{\left(u_{1}, u_{2},-u_{2}, u_{1}, u_{2},-u_{1},-u_{1},-u_{2}\right) \mid u_{1}, u_{2} \in \mathbb{R}\right\} .
$$

The $\mathbb{Z}_{4}$-symmetric periodic orbits bifurcating from the equivariant Hopf point are shown in Figures 5 and 6 . These solutions have already been computed in [3] by a Galerkin method based on Fourier modes.

Using the methods of Section 3, SYMPERCON finds that this branch of $\mathbb{Z}_{4}$-symmetric periodic orbits undergoes a flip pitchfork bifurcation at $\lambda^{*}=2.6738$. A point $x^{*}$ on the periodic orbit at the symmetry breaking bifurcation point is given by

$$
x^{*}=(1.7041,3.0132,0.86523,4.8152,3.6590,1.5591,0.92057,6.0440),
$$

and the period of this periodic solution is $T^{*}=5.0410$. The $\mathbb{Z}_{4}$-periodic solution close to the symmetry breaking bifurcation (at the parameter $\lambda=2.7904$ ) and a $\mathbb{Z}_{2}$-symmetric periodic orbit after the bifurcation (at the parameter $\lambda=2.6734$ ) can be seen in Figure 8, the bifurcation diagram is shown in Figure 7. 


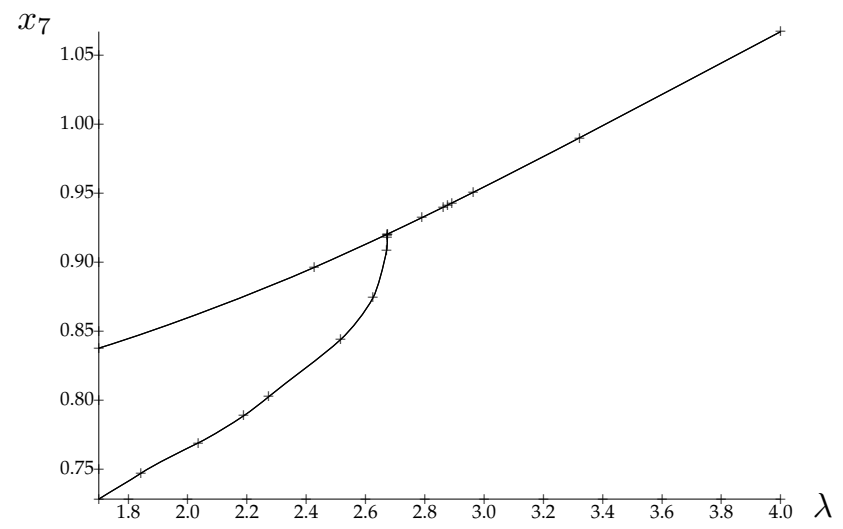

Figure 7: Flip pitchfork bifurcation from $\mathbb{Z}_{4}$ to $\mathbb{Z}_{2}$-symmetry in the 4-cell Brusselator
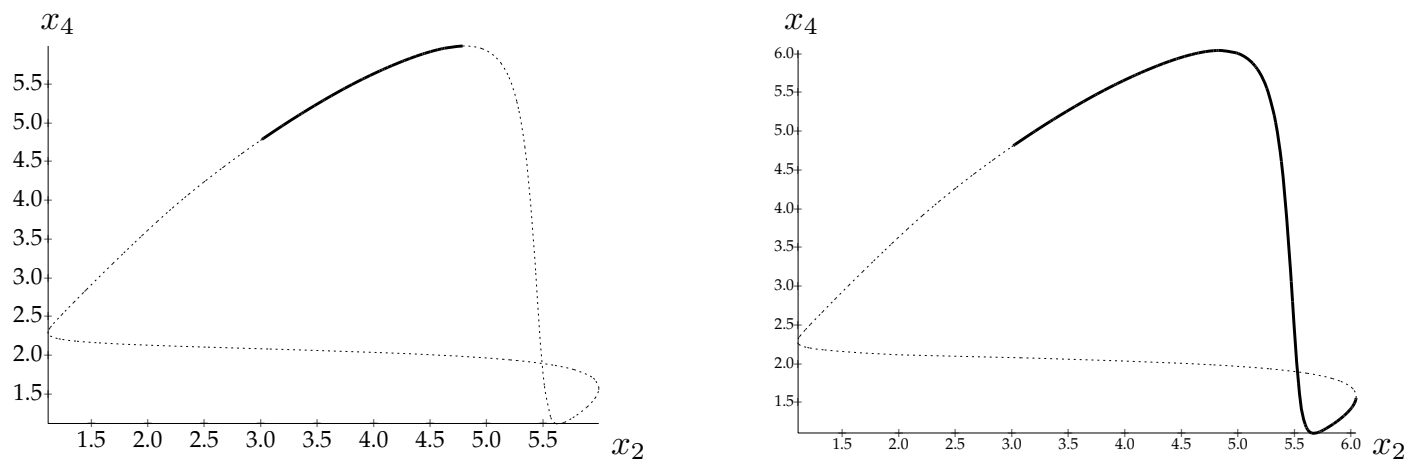

Figure 8: Symmetric periodic orbits of the 4-cell Brusselator before and after a flip pitchfork bifurcation from $\mathbb{Z}_{4}$-symmetry to $\mathbb{Z}_{2}$-symmetry - the bold parts of the trajectories are computed numerically.

\subsection{Electronic ring oscillator}

In this section we a simulate an electrical circuit consisting of $n$ MOSFET-inverters taken from Kampowsky, Rentrop, Schmidt [15]. Such a circuit can be modelled in the following way: let $U_{i}$ be the voltage at the $i$-th node, then using Kirchhoff's law we get the differential equations

$$
f, \tilde{f}: \mathbb{R}^{n} \rightarrow \mathbb{R}^{n}, \quad \dot{U}=f(U), \quad f(U)=-C^{-1} \tilde{f}(U)
$$

for the vector $U=\left(U_{1}, \ldots, U_{n}\right)$ of the voltages. Here $C$ is the capacity matrix

$$
C=\left(\begin{array}{cccc}
2 C_{p}+C & -C_{p} & & -C_{p} \\
-C_{p} & 2 C_{p}+C & -C_{p} & \\
& \ddots & \ddots & \\
-C_{p} & & -C_{p} & 2 C_{p}+C
\end{array}\right),
$$




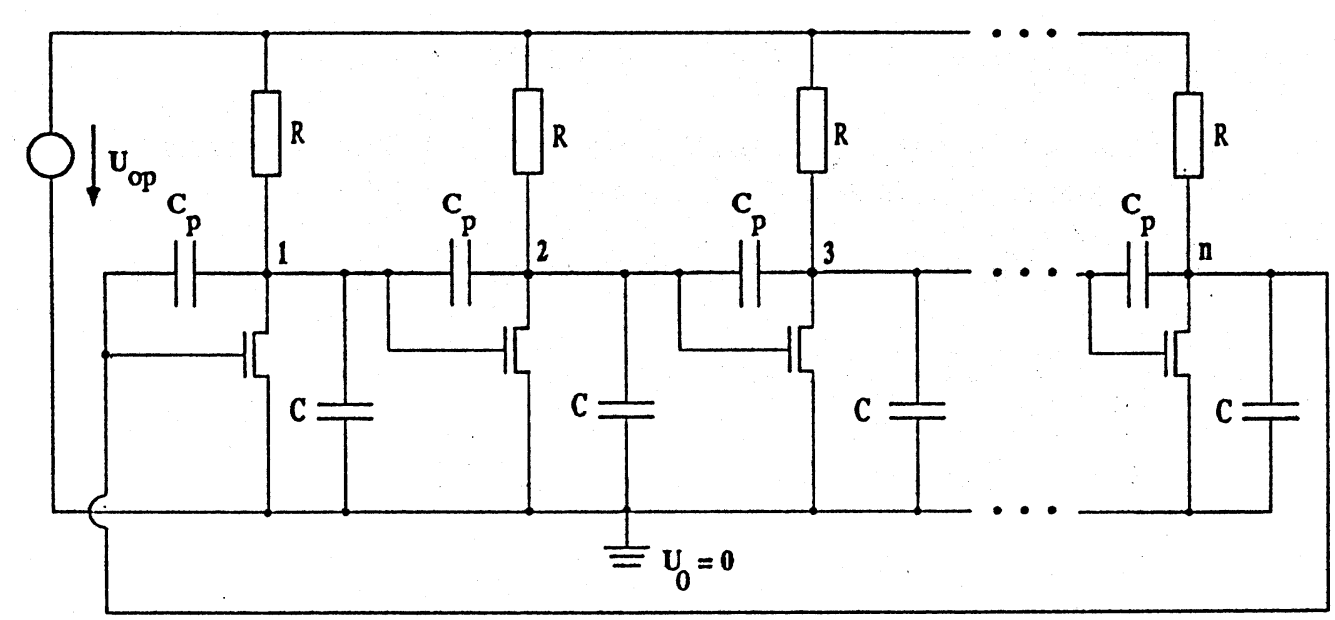

Figure 9: Circuit of ring oscillator

and the function $f$ is given by

$$
\tilde{f}(U)=\left(\begin{array}{c}
1 / R\left(U_{1}-U_{\mathrm{op}}\right)+g\left(U_{n}, U_{1}, U_{0}\right) \\
1 / R\left(U_{2}-U_{\mathrm{op}}\right)+g\left(U_{1}, U_{2}, U_{0}\right) \\
\vdots \\
1 / R\left(U_{n}-U_{\mathrm{op}}\right)+g\left(U_{n-1}, U_{n}, U_{0}\right)
\end{array}\right),
$$

where

$$
\begin{aligned}
g\left(U_{G}, U_{D}, U_{S}\right)= & K \max \left\{\left(U_{G}-U_{S}-U_{T}\right), 0\right\}^{2} \\
& -K \max \left\{\left(U_{G}-U_{D}-U_{T}\right), 0\right\}^{2} .
\end{aligned}
$$

The constants $K, U_{T}, U_{0}, U_{\mathrm{op}}, R$ and the capacities $C$ and $C_{p}$ are technical parameters, which have (after appropriate scaling) the values $K=0.2, U_{T}=1, U_{0}=0, U_{\mathrm{op}}=5, R=5$, $C=0.21, C_{p}=5 \cdot 10^{-3}$, see [15]. From [15] we took the initial values of a periodic solution, namely $U_{1}=4.2087, U_{2}=0.917, U_{3}=2.6534, U_{4}=4.2762, U_{5}=0.6928, T=6.298$. This periodic solution is shown in Figure 10. Furthermore there is a stationary solution given by $U_{i}(t) \equiv 2.56155, i=1, \ldots, 5$. Our aim is now the numerical simulation of oscillations of large electrical circuits, i.e., the computation of periodic orbits for large $n$.

The function $f: \mathbb{R}^{n} \rightarrow \mathbb{R}^{n}$ is $\mathbb{Z}_{n}$-equivariant where the generating element $\gamma_{n}$ of

$$
\mathbb{Z}_{n}=\left\{\mathrm{id}, \gamma_{n}, \ldots \gamma_{n}^{n-1}\right\}
$$

is acting on $\mathbb{R}^{n}$ by shifting the components to the right

$$
\gamma_{n}\left(x_{1}, \ldots, x_{n}\right)=\left(x_{2}, \ldots, x_{n}, x_{1}\right) .
$$

This representation of $\mathbb{Z}_{n}$ is called the regular representation of $\mathbb{Z}_{n}$, see [21]. Using SYMPERCON we checked that the above periodic solution (with $n=5$ ) has spatio-temporal symmetry $L=\mathbb{Z}_{5}$. Since the stationary solution is $\mathbb{Z}_{n}$-invariant the periodic solution might have branched from the stationary solution via an equivariant Hopf bifurcation. So we introduce $U_{\text {op }}$ as a 


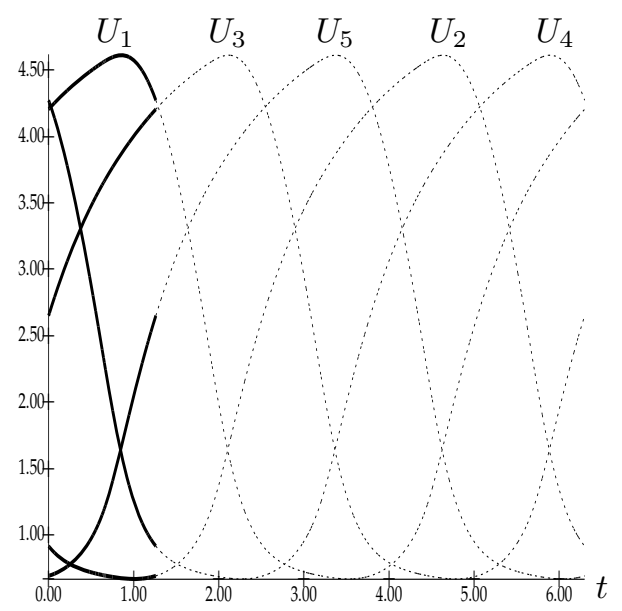

Figure 10: Periodic solution for 5 inverters

continuation parameter and examine the stationary $\mathbb{Z}_{n}$-invariant solutions $U=(u, \ldots, u) \in \mathbb{R}^{n}$, $u \in \mathbb{R}$, which are given by the equation

$$
\begin{aligned}
& 1 / R\left(u-U_{\mathrm{op}}\right)+g\left(u, u, U_{0}\right)=0 \\
& \Longleftrightarrow \quad 1 / R\left(u-U_{\mathrm{op}}\right)+K\left(\max \left(u-U_{T}, 0\right)\right)^{2}=0 .
\end{aligned}
$$

In $U=(u, \ldots, u)$ the Jacobian $\mathrm{D}_{U} f(U)$ is $\mathbb{Z}_{n}$-symmetric:

$$
\gamma \mathrm{D}_{U} f(U)=\mathrm{D}_{U} f(U) \gamma \quad \forall \gamma \in \Gamma=\mathbb{Z}_{n}
$$

From elementary representation theory it is known that in a regular representation each irreducible representation $\vartheta_{i}$ is contained $n_{i}$ times, where $n_{i}$ is the dimension of the representation $\vartheta_{i}$, see [21]. Finite cyclic groups $\mathbb{Z}_{n}$ only have two dimensional complex irreducible representations (as defined in Remark 2.4). Over $\mathbb{C}$ they are given by

$$
\vartheta_{j}\left(\gamma_{n}\right)=e^{\frac{2 \pi \mathrm{i} j}{n}}, \quad j=0, \ldots n-1,
$$

see [21]. Let $X_{j}$ denote the $\Gamma$-irreducible subspace of $\mathbb{R}^{n}$ belonging to $\vartheta_{j}$. Then $X_{j}, j=$ $0, \ldots, n-1$, is spanned by the real part $v_{j}$ and imaginary part $w_{j}$ of the vector

$$
v_{j}+\mathrm{i} w_{j}=\left(1, e^{\frac{2 \pi \mathrm{i} j}{n}}, e^{\frac{2 \pi \mathrm{i} 2 j}{n}}, \ldots, e^{\frac{2 \pi \mathrm{i}(n-1) j}{n}}\right) .
$$

Using (5.2) we conclude that the Jacobian $\mathrm{D}_{U} f(U)$ can be diagonalised over $\mathbb{C}$. Here $v_{j}+\mathrm{i} w_{j}$ is an eigenvector to the eigenvalue

$$
\lambda_{j}=\frac{1 / R+2 K \max \left(u-U_{T}, 0\right) e^{\frac{2 \pi \mathrm{i}(n-1) j}{n}}}{C+\left(2-2 \cos \frac{2 \pi j}{n}\right) C_{p}},
$$

as can be computed easily. Setting $\operatorname{Re}\left(\lambda_{j}\right)=0$ we get simple expressions for the stationary solution $u=u^{(j)}$ and the corresponding parameter $U_{\mathrm{op}}^{(j)}$ for which an equivariant Hopf bifurcation can occur, and also for the Hopf frequency $\omega_{j}$. Using the equivariant Hopf Theorem 4.5 we conclude that every bifurcating periodic solution has the symmetry $L=\mathbb{Z}_{n}$. But we also have 
Table 1: Amount of work for the computation of symmetric periodic orbits with trivial isotropy of the electrical circuit depending on the number $n$ of inverters

\begin{tabular}{l|c|c|} 
& $\begin{array}{l}\text { with exploitation } \\
\text { of symmetry }\end{array}$ & $\begin{array}{c}\text { without exploita- } \\
\text { tion of symmetry }\end{array}$ \\
\hline flow $\Phi_{t}(x)$ & $O(n)$ & $N_{\text {step }} * O(n)$ \\
Wronskian matrix $\mathrm{D}_{x} \Phi_{t}(x)$ & $O(n)$ & $N_{\text {step }} * O\left(n^{2}\right)$ \\
linear algebra & $O(n)$ & $O\left(n^{3}\right)$
\end{tabular}

to determine the homomorphism $\Theta$ of the bifurcating periodic orbits and thereby their isotropy $K$ and drift symmetry $\alpha$. For this we have to know the operation of $\Gamma \times S^{1}$ on the Hopf eigenspace $N_{\omega^{*}}=X_{j}$, see (4.6). We compute that $\Gamma \times S^{1}$ operates on the irreducible subspace $X_{j}=\operatorname{span}\left(v_{j}, w_{j}\right)$ in the following way

$$
(\gamma, \theta)\left(v_{j}+\mathrm{i} w_{j}\right)=\vartheta_{j}(\gamma) e^{2 \pi \mathrm{i} \theta}\left(v_{j}+\mathrm{i} w_{j}\right),
$$

where $\gamma \in \Gamma, \theta \in S^{1} \equiv \mathbb{R} / \mathbb{Z}$. We can characterize the homomorphism $\Theta_{j}$ of the bifurcating periodic orbits tangential to $N_{\omega^{*}}=X_{j}$ by the homomorphism $\Theta_{j}\left(\gamma_{n}\right)$ applied to the generating element $\gamma_{n}$ of $\mathbb{Z}_{n}$. From (5.5) and (5.3) we get

$$
\Theta_{j}\left(\gamma_{n}\right)=-j / n
$$

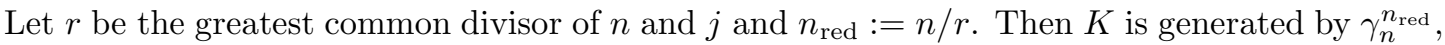
and the fixed point space $\operatorname{Fix}(K)$ contains all vectors in $\mathbb{R}^{n}$ which consist of $r$ equal sections of length $n_{\text {red }}$. Therefore $n_{\text {red }}$ is the dimension of the reduced system.

After restriction onto the fixed point space $\operatorname{Fix}(K)=\mathbb{R}^{n_{\text {red }}}$ we obtain $L=\mathbb{Z}_{n_{\text {red }}}, K=\{$ id $\}$. Then $\gamma_{n_{\text {red }}} \equiv \gamma_{n} K$ is a generating element of $\mathbb{Z}_{n_{\text {red }}}$. The group $\mathbb{Z}_{n_{\text {red }}}$ also acts on $\mathbb{R}^{n_{\text {red }}}$ by cyclically permuting the components of every $x \in \mathbb{R}^{n_{\text {red }}}$. The representation $\vartheta_{j_{\text {red }}}$ with $j_{\text {red }}:=$ $j / r$ is the irreducible representation of $\mathbb{Z}_{n_{\text {red }}}$ on $\mathbb{R}^{n_{\text {red }}}$ which corresponds to $\vartheta_{j}$. Thus,

$$
N_{\omega^{*}}^{\mathrm{red}}=\left\langle V_{j_{\mathrm{red}}}, W_{j_{\mathrm{red}}}\right\rangle \subset \mathbb{R}^{n_{\mathrm{red}}}
$$

is the initial plane for the periodic solutions in the reduced coordinates. Finally we have to determine the element $\alpha \in \mathbb{Z}_{n_{\text {red }}}$ with the smallest phase shift $\Theta_{\text {red }}(\alpha)$. Let $\alpha=\gamma_{n_{\text {red }}}^{k}$, where $k$ is an integer between 1 and $n_{\text {red }}-1$. Then $\alpha$ is given by the equation $\Theta_{\text {red }}(\alpha)=1 / n_{\text {red }}$, i.e.,

$$
\vartheta_{j_{\text {red }}}(\alpha) e^{2 \pi \mathrm{i} / n_{\text {red }}}=1 \Leftrightarrow j_{\text {red }} k \equiv 1 \quad\left(\bmod n_{\text {red }}\right) .
$$

By the just described method we can compute initial values for periodic solutions for arbitrary $n$, and by exploiting symmetry we can reduce the amount of work effectively: if the considered periodic solution has large spatial symmetry then the ODE is reduced to a system with small dimension $n_{\text {red }}$. For example, in the case $n=1000$, there is a periodic solution with $K=\mathbb{Z}_{200}$, $n_{\text {red }}=5$, which corresponds to the above computed solution with $n=5$.

If the periodic solution does not have any spatial symmetry, but only spatio-temporal symmetry then only the integration interval can be reduced by exploitation of symmetry. Let $N_{\text {step }}$ be the number of integration steps for the full integration interval $[0, T]$. If the symmetry is not exploited, $N_{\text {step }} * O(n)$ multiplications and divisions are needed for the computation of the flow; exploiting the symmetry we can reduce the number of integration steps from $N_{\text {step }}$ to $N_{\text {step }} / n$, 
since the integration interval is only $[0, T / n]$. Hence for large $n$ the number of time steps is $O(1)$, and so the number of multiplications and divisions for the computation of the flow is $O(n)$ when symmetry is exploited. Since the derivatives $\mathrm{D}_{x} f(x)$ are tridiagonal matrices and hence contain only $O(n)$ non-zero components, $N_{\text {step }} * O\left(n^{2}\right)$ multiplications are needed for the computation of the Wronskian matrices $\mathrm{D}_{x} \Phi_{t}(x)$ by integration of the variational equation if the symmetry is not exploited; if the symmetry is exploited then for large $n$ only few steps of integration are necessary for the computation of the Wronskian matrices. Therefore the Wronskian matrices are sparse, and only contain non-zero entries close to the diagonal. Hence by exploitation of symmetry the amount of work for the computation of the Wronskian reduces to $O(n)$. For the solution of the linear equations which arise in the Gauss-Newton method $O\left(n^{3}\right)$ multiplications are needed if the symmetry is not exploited, and the amount of work for the linear algebra reduces to $O(n)$ due to the sparse structure of the Wronskians if the symmetry is exploited. Altogether we obtain a remarkable reduction of the computational cost by exploitation of symmetry, c.f. Table 1.

\section{Conclusion and future directions}

In this paper we have presented efficient algorithms for the computation of generic symmetry changing but isotropy preserving bifurcations of periodic orbits in systems where the symmetry group is discrete. These bifurcations were analyzed by Golubitsky et al (equivariant Hopf bifurcation, see eg [11]) and Fiedler (flip doubling and flip pitchfork bifurcations, see [9]). General symmety breaking bifurcations of periodic orbits in systems with discrete symmetry group were classified by Lamb and Melbourne [18], see also [19] and [24] for the case of continuous symmetry groups. In future work we will extend our numerical results to compute symmetry changing bifurcations of periodic orbits of arbitrary Lie group actions. In the paper [26] we extend the methods presented here to Hamiltonian systems with continuous symmetries building on the persistence results of $[25]$.

\section{References}

[1] W.-J. Beyn, A. Champneys, E. Doedel, W. Govaerts, Y. A. Kuznetsov, B. Sandstede. Numerical continuation, and computation of normal forms. Chapter 4 of Handbook of Dynamical Systems, vol. 2, B. Fiedler ed., pp. 149-219, 2002.

[2] K. A. Cliffe, A. Spence, and S. J. Tavener. O(2)-symmetry breaking bifurcation: with application to the flow past a sphere in a pipe. Internat. J. Numer. Methods Fluids 32(2): 175-200, 2000.

[3] M. Dellnitz. A computational method and pathfollowing for periodic solutions with symmetry. In D. Roose, B. de Dier, A.Spence, ed., Continuation and Bifurcations: Numerical Techniques and Applications, NATO ASI SERIES C, vol. 313, pp. 153-167, 1990.

[4] M. Dellnitz. Computational bifurcation of periodic solutions in systems with symmetry. IMA Journal of Numerical Analysis, 12: 429-455, 1992.

[5] P. Deuflhard. Computation of periodic solutions of nonlinear ODE's. BIT 24: 456-466, 1984.

[6] P. Deuflhard. Recent progress in extrapolation methods for ordinary differential esquations. SIAM Review 27: 505-535, 1985. 
[7] P. Deufhard, B. Fiedler, P. Kunkel. Efficient numerical pathfollowing beyond critical points. SIAM J. Numer. Anal., 18: 949-987, 1987.

[8] E.J. Doedel, R.C. Paffenroth, A.R. Champneys, T.F. Fairgrieve, Y.A. Kuznetsov, B.E. Oldeman, B. Sandstede and X.-J. Wang. AUTO2000: Continuation and bifurcation software for ordinary differential equations. Technical Report, Concordia University 2000, available from http://www.cs.concordia.ca.

[9] B. Fiedler. Global Bifurcation of Periodic Solutions with Symmetry. Lecture Notes in Mathematics. Springer Verlag, Berlin, Heidelberg, New York, 1988.

[10] K. Gatermann, A. Hohmann. Symbolic exploitation of symmetry in numerical pathfollowing. IMPACT Comput. Sci. Engrg. 3: 330-365, 1991.

[11] M. Golubitzky, I. Stewart, D. Schaeffer. Singularities and Groups in Bifurcation Theory, volume 2. Springer-Verlag, Berlin, Heidelberg, New York, 1988.

[12] A. Griewank and G. W. Reddien. The calculation of Hopf points by a direct method. IMA J. Numer. Anal. 3: 295-303, 1983.

[13] J. Guckenheimer and P. Holmes. Nonlinear Oscillations, Dynamical Systems, and Bifurcations of Vector Fields, Applied Mathematical Sciences, vol. 42. Springer-Verlag, New York, Heidelberg, Berlin, 1990.

[14] A. D. Jepson. Numerical Hopf Bifurcation. PhD thesis, California Institute of Technology, 1981.

[15] W. Kampowsky, P. Rentrop, W. Schmidt. Classification and numerical simulation of electric circuits. Surveys on Mathematics for Industry, 2(1): 23-65, 1992.

[16] Y. Kuznetsov. Elements of Applied Bifurcation Theory. Applied Mathematical Sciences, vol. 112. Springer, New York, 1995.

[17] Y.A. Kuznetsov and V.V. Levitin. CONTENT: A multiplatform environment for continuation and bifurcation analysis of dynamical systems. Centrum voor Wiskunde en Informatica 1997, available from ftp://ftp.cwi.nl/pub/CONTENT.

[18] J. S. W. Lamb and I. Melbourne. Bifurcation from discrete rotating waves. Archive Rat. Mech. Anal. 149: 229-270, 1999.

[19] J. Lamb, I. Melbourne, C. Wulff. General bifurcations from periodic solutions with spatiotemporal symmetry, including mode interactions and resonances. J. Differential Equations, 191(2): 377-407, 2003.

[20] A. Schebesch. SYMPERCON - a package for the numerical continuation of symmetric periodic orbits. Diplomarbeit, Freie Universität Berlin, 2004. The current version of SYMPERCON can be obtained from http://www.maths.surrey.ac.uk/personal/st/C.Wulff/.

[21] J.-P. Serre. Linear Representations of Finite Groups. Springer-Verlag, New York, Heidelberg, Berlin, 1977.

[22] C. Wulff. Numerische Pfadverfolgung von periodischen Lösungen mit Symmetrie. Diplomarbeit, Freie Universität Berlin, 1993.

[23] C. Wulff, A. Hohmann, P. Deufhard. Numerical continuation of periodic orbits with symmetry. Technical report, Konrad-Zuse-Zentrum, Berlin, 1994. 
[24] C. Wulff, J. Lamb, I. Melbourne. Bifurcation from relative periodic solutions. Ergodic Th. Dyn. Syst. 21: 605-635, 2001.

[25] C. Wulff. Persistence of Hamiltonian relative periodic orbits. J. Geom. Phys. 48: 309-338, 2003.

[26] C. Wulff and A. Schebesch. Numerical continuation of Hamiltonian relative periodic orbits. Preprint. 